Series A

I. MATHEMATICA

588

\title{
WHITEHEAD TORSION AND GROUP ACTIONS
}

BY

SÖREN ILLMAN

HELSINKI 1974

S U O M A L A I N N TIEDEAKATEMIA 
Copyright (C) 1974 by Academia Scientiarum Fennica ISSN 0066-1953

ISBN 951-41-0202-9

Communicated 11 March 1974 


\section{Contents}

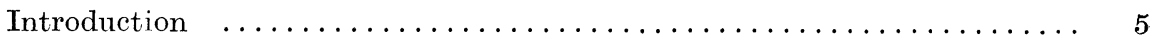

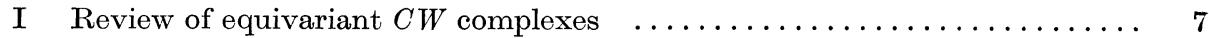

II Foundations of equivariant simple-homotopy theory $\ldots \ldots \ldots \ldots \ldots \ldots \ldots 11$

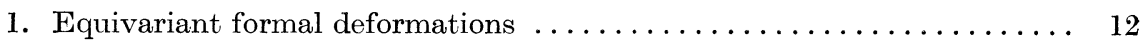

2. The equivariant Whitehead group $W h_{G}(X) \quad \ldots \ldots \ldots \ldots \ldots \ldots \ldots$

3. Geometric equivariant Whitehead torsion of a $G$-homotopy equivalence 21

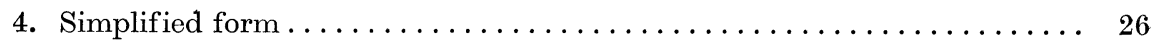

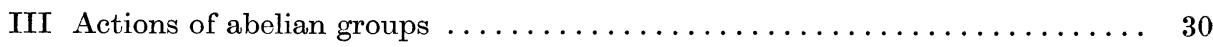

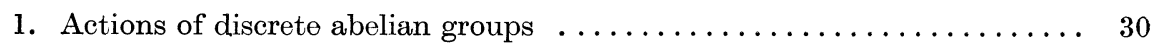

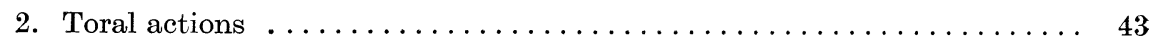




\section{Introduction}

Let $G$ be a discrete group or a compact Lie group, and let $X$ and $Y$ denote finite equivariant $C W$ complexes. In Section 1 of Chapter II we introduce the notion of an equivariant simple-homotopy equivalence $f: X \rightarrow Y$. The main part for this definition is the definition of an equivariant elementary expansion, see Definition 1.1 in Chapter II. Having defined equivariant elementary expansions (collapses) the notions, equivariant formal deformation, equivariant expansion (collapse), and equivariant simple-homotopy equivalence, follow in complete analogy with the corresponding notions in the ordinary non-equivariant case as defined by Whitehead [18].

We then define an equivariant Whitehead group $W h_{G}(X)$ of any finite equivariant $C W$ complex $X$. If $G$ is the trivial group, and $X$ thus is an ordinary $C W$ complex, this simply gives us the geometrically defined Whitehead group $W h(X)$ of $X$ which, for connected $X$, is known to be isomorphic to $W h\left(\pi_{1}(X)\right)$, the (algebraicly defined) Whitehead group of the group $\pi_{1}(X)$, see Cohen [6], Eckmann-Maumary [1], and Stöcker [15]. We prove that if $G$ acts freely on $X$ then $W h_{G}(X) \cong$ $W h(G \backslash X)$. Thus if $G$ is a discrete group acting freely on $X$, and $X$ is simply connected, we have $W h_{G}(X) \cong W h(G)$. In Section 1 of Chapter III we determine $W h_{G}(X)$ in terms of Whitehead groups of quotient groups of subgroups of $G$, for the case when $G$ is a discrete abelian group and $X$ is such that for any subgroup $H$ of $G$ each component of $X^{H}$ is simply connected.

Let now again $G$ denote any discrete group or an arbitrary compact Lie group. In Section 3 of Chapter II we define the geometric equivariant Whitehead torsion $\tau_{g}(f) \in W h(X)$ of any $G$-homotopy equivalence $f: X \rightarrow Y$, and prove that $f$ is an equivariant simple-homotopy equivalence if and only if $\tau_{g}(f)=0$. If $G$ acts freely on $X$ and $Y$, then any $G$-homeomorphism $f: X \rightarrow Y$ is an equivariant simple-homotopy equivalence. This follows from the recent affirmative answer by $T$. Chapman to the question of topological invariance of ordinary Whitehead torsion, and the isomorphism $W h_{G}(X) \cong W h(G \backslash X)$ for free actions. We also give a sum theorem for $\tau_{g}(f)$. In Section 4 we prove a technical result which says that any element in $W h_{G}(X)$ can be represented by 
an element which is in "simplified form», see Corollary 4.4 in Chapter II for the precise statement. This result is the key to the results proved in Chapter III.

In Chapter III we first consider actions of discrete abelian groups and prove the already mentioned theorem about $W h_{G}(X)$, see Theorem 1.4 and the discussion preceeding it. Using known results about Whitehead groups of groups this gives us information about $W h_{G}(X)$. For example it follows that if $G=Z_{m}, m \geq 1$, is a finite cyclic group, and $X$ is such that each component of any $X^{H}, H \subset G$, is simply connected, then $W h_{G}(X)$ is a free abelian group of finite rank. If $G=Z_{2}, Z_{3}, Z_{4}$ or $Z_{6}$, then $W h_{G}(X)=0$, where $X$ is as before, and hence in this situation any $G$-homotopy equivalence is an equivariant simple-homotopy equivalence. We also say something about the case $G=Z \oplus \ldots \oplus Z$. We conclude the first section by an example of an inclusion $i: X \rightarrow W$, in fact $X=\{x\}$, of equivariant $C W$ complexes which is a G-homotopy equivalence but not an equivariant simple-homotopy equivalence and the induced inclusion on the orbit spaces is an ordinary simple-homotopy equivalence and moreover if we forget about the $G$-action then the inclusion $i: X \rightarrow W$ is an ordinary simple-homotopy equivalence.

In the final section we consider actions by an $n$-dimensional torus $G=T^{n}, n \geq 1$. In this case we have $W h_{G}(X)=0$, for every $X$ satisfying the condition that for any closed subgroup $H$ of $G=T^{n}$ each component of $X^{H}$ is simply connected, and hence in this situation any $G$-homotopy equivalence is an equivariant simple-homotopy equivalence. This result applies in particular to differentiable $T^{n}$ actions on compact differentiable manifolds.

Notations. By $I^{n}$ we denote the $n$-fold product of the unit interval with itself and $I^{n-1}$ is identified with the front $(n-1)$-face $I^{n-1} \times\{0\} \subset I^{n}$. By $J^{n-1}$ we denote the union of all other $(n-1)$-faces and $\partial I^{n}$ is the boundary of $I^{n}$, i.e. $\partial I^{n}=I^{n-1} \cup J^{n-1}$. We shall use $G$-spaces of the form $G / H \times I^{n}$, where $H$ is some closed subgroup of $G$. Here $G$ acts trivially on $I^{n}$ and by the standard left action on $G / H$. If $H$ is a closed subgroup of $G$ we denote by $(H)$ the family of all subgroups conjugate to $H$, and for any $G$-space $X$, we denote by $X^{H}$ the set of points fixed under $H$. 


\section{Chapter I. Review of equivariant CW complexes}

In this chapter $G$ denotes a topological group which is either a compact Lie group or a discrete group.

Definition 1.1. Let $X$ be a Hausdorff $G$-space and $A$ a closed $G$ subset of $X$, and $n$ a non-negative integer. We say that $X$ is obtainable from $A$ by adjoining equivariant $n$-cells if there exists a collection $\left\{c_{j}^{n}\right\}_{j \in J}$ of closed $G$-subsets of $X$ such that

1) $X=A \cup\left(\bigcup_{j \in J} c_{j}^{n}\right)$, and $X$ has the topology coherent with $\left\{A, c_{j}^{n}\right\}_{j \in J}$.

2) Denote $\dot{c}_{j}^{n}=c_{j}^{n} \cap A$, then

$$
\left(c_{j}^{n}-\dot{c}_{j}^{n}\right) \cap\left(c_{i}^{n}-\dot{c}_{i}^{n}\right)=\varnothing \text { if } i \neq j .
$$

3) For each $j \in J$ there exists a closed subgroup $H_{j}$ of $G$ and a $G$-map

$$
f_{j}:\left(G / H_{j} \times I^{n}, G / H_{j} \times \partial I^{n}\right) \rightarrow\left(c_{j}^{n}, \dot{c}_{j}^{n}\right)
$$

such that $f_{j}\left(G / H_{j} \times I^{n}\right)=c_{j}^{n}$, and $f_{j}$ maps $G / H_{j} \times\left(I^{n}-\partial I^{n}\right) G$-homeomorphically onto $c_{j}^{n}-\dot{c}_{j}^{n}$.

Such a $G$-pair $(X, A)$ in fact determines the $G$-subsets $c_{j}^{n}$ uniquely; that is, any two collections of closed $G$-subsets of $X$ which satisfy condition 1)-3) in the above definition are the same. Moreover $X$ is not obtainable from $A$ by adjoining equivariant $m$-cells if $m \neq n$. We call the $G$-subsets $c_{j}^{n}$ for the equivariant $n$-cells of $(X, A)$ and also say that $X$ is obtained from $A$ by adjoining the equivariant $n$-cells $c_{j}^{n}$. The $G$ subsets $b_{j}^{n}=c_{j}^{n}-\dot{c}_{j}^{n}$, which are open subsets of $X-\mathrm{A}$, are called open equivariant $n$-cells of $(X, A)$. Any $G$-map $f_{j}: G / H_{j} \times I^{n} \rightarrow c_{j}^{n}$ which satisfies the conditions in Definition 1.1 is called a characteristic $G$-map for $c_{j}^{n}$, and its restriction $f_{j} \mid: G / H_{j} \times \partial I^{n} \rightarrow \dot{c}_{j}^{n} \rightarrow A$ is called an attaching $G$-map for $c_{j}^{n}$. We call $\left(H_{j}\right)$ for the type of $c_{j}^{n}$.

Definition 1.2. An equivariant relative $C W$ complex $(X, A)$ consists of a Hausdorff $G$-space $X$, a closed $G$-subset $A$ of $X$, and an increasing filtration of $X$ by closed $G$-subsets $(X, A)^{k}, k=0,1, \ldots$, such that 
1) $(X, A)^{0}$ is obtainable from $A$ by adjoining equivariant 0 -cells, and for $k \geq 1 \quad(X, A)^{k}$ is obtainable from $(X, A)^{k-1}$ by adjoining equivariant $k$-cells.

2) $X=\bigcup_{k \geq 0}(X, A)^{k}$, and $X$ has the topology coherent with $\left\{(X, A)^{k}\right\}_{k \geq 0}$.

The $G$-subset $(X, A)^{k}$ is called the $k$-skeleton of $(X, \mathrm{~A})$. Observe that it is part of the structure of an equivariant relative $C W$ complex $(X, A)$. The (open) equivariant $k$-cells of $\left((X, A)^{k},(X, A)^{k-1}\right)$ are called (open) equivariant $k$-cells of $(X, A)$. Observe that the orbit space pair $(G \backslash X, G \backslash A)$ inherits the structure of an ordinary relative $C W$ complex with $k$-skeleton equal to $G \backslash(X, A)^{k}$. We say that $\operatorname{dim}(X, A)=m$ if $X=(X, A)^{m}$ but $X \neq(X, A)^{m-1}$. If no such integer $m$ exists we say that $\operatorname{dim}(X, A)=\infty$. We have $\operatorname{dim}(X, A)=\operatorname{dim}(G \backslash X, G \backslash A)$. If $(X, A)$ is a $G$-pair which admits the structure of an equivariant relative $C W$ complex then $\operatorname{dim}(X, A)$ is well-defined, that is, does not depend on the skeleton filtration. This follows since the corresponding statement for ordinary relative $C W$ structures is a well-known fact.

Let $(X, A)$ be an equivariant relative $C W$ complex, and let $X_{0}$ be a closed $G$-subset of $X$. Then we say that $\left(X_{0}, X_{0} \cap A\right)$ is a subcomplex of $(X, A)$ if the filtration $X_{0} \cap(X, A)^{k}, k=0,1, \ldots$, gives $\left(X_{0}, X_{0} \cap A\right)$ the structure of an equivariant relative $C W$ complex. It is easy to show that then $\left(X, X_{0} \cup A\right)$ is an equivariant relative $C W$ complex with skeletons $\left(X, X_{0} \cup A\right)^{k}=X_{0} \cup(X, A)^{k}$. In fact $X_{0} \cup(X, A)^{k}$ is obtained from $X_{0} \cup(X, A)^{k-1}$ by adjoining all the equivariant $k$-cells of $(X, A)$ which are not equivariant $k$-cells of $\left(X_{0}, X_{0} \cap A\right)$.

If $A=\varnothing$ we call $X$ an equivariant $C W$ complex and denote its $k$-skeleton by $X^{k}$. An equivariant $C W$ pair $\left(X, X_{0}\right)$ consists of an equivariant $C W$ complex $X$ and a subcomplex $X_{0}$ of $X$. An equivariant $C W$ complex $X$ (pair $\left(X, X_{0}\right)$ ) is said to be finite if $X$ has only a finite number of equivariant cells. Observe that in case $G$ is a discrete group and $X$ thus also automatically has the structure of an ordinary $C W$ complex this does not mean that the ordinary $C W$ complex $X$ necessarily is finite.

Let $(X, A)$ be a $G$-equivariant relative $C W$ complex and $(Y, B)$ a $G^{\prime}$-equivariant relative $C W$ complex. Assume that either both $X$ and $Y$ are locally compact or $X$ is arbitrary and $Y$ is compact. Then $(X, A) \times(Y, B)=(X \times Y, X \times B \cup A \times Y)$ is a $\left(G \times G^{\prime}\right)$-equivariant relative $C W$ complex with $n$-skeleton equal to $\bigcup_{k+p=n}(X, A)^{k} \times(Y, B)^{p}$.

In this paper we shall only use this in the case $G^{\prime}=\{e\}$, that is, $(Y, B)$ 
is an ordinary relative $C W$ complex and the product $(X, A) \times(Y, B)$ is again a $G$-equivariant relative $C W$ complex.

The following result is not explicitly stated in Chapter I of [10] although it is an immediate consequence of results given there. We shall have use of it in this paper and hence we state it here and give its proof.

Proposition 1.3. Let $(X, A)$ be an equivariant relative $C W$ complex. Then the inclusion $i: A \rightarrow X$ is a $G$-homotopy equivalence if and only if $A$ is a strong $G$-deformation retract of $X$.

Proof. Assume that $i: A \rightarrow X$ is a $G$-homotopy equivalence. Then it follows that for each closed subgroup $H$ of $G$ the inclusion $i \mid: A^{H} \rightarrow X^{H}$ is a homotopy equivalence. Thus the pair $\left(X^{H}, A^{H}\right)$ is $n$-connected for all $n$. By Corollary 1.11 in Chapter $\mathrm{I}$ of $[10]$ this imples that $(X, A)$ is equivariantly $n$-connected for all $n$ (see Definition 1.9. in Chapter I of [10]). Thus, by Corollary 2.9 in Chapter I of [10], any G-map $f:(X, A) \rightarrow(X, A)$ is $G$-homotopic rel. $A$ to a $G$-map from $X$ into $A$. Applying this to the identity map we see that $A$ is a strong $G$-deformation retract of $X$. The sif» part of the claim is obvious.

Now let $G^{\prime}$ be another topological group which is either a compact Lie group or a discrete group, and let $\phi: G \rightarrow G^{\prime}$ be a continuous homomorphism. Let $(X, A)$ be a $G$-equivariant relative $C W$ complex and $(Y, B)$ a $G^{\prime}$-equivariant relative $C W$ complex. A $\varphi$-map $f:(X, A) \rightarrow(Y, B)$, i.e. $f(g x)=\varphi(g) f(x) \quad x \in X, g \in G$, is called skeletal if $f\left((X, A)^{k}\right) \subset(Y, B)^{k}$, for all $k \geq 0$. Observe that if $X$ and $Y$ in fact are $G$-equivariant and $G^{\prime}$-equivariant, respectively, $C W$ complexes and $A$ and $B$ are subcomplexes then the above condition reads $f\left(X^{k} \cup A\right) \subset Y^{k} \cup B$, for all $k \geq 0$, and hence the absolute map $f: X \rightarrow Y$ need not itself be skeletal. This "freedom" in the definition of a skeletal map between pairs is in some cases extremely convenient. The following theorem is Theorem 2.14 in Chapter I of [10].

Theorem 1.4. Let $(X, A)$ and $(Y, B)$ be a $G$-equivariant and a $G^{\prime}$-equivariant, respectively, relative $C W$ complex. Assume that the $\varphi$-map $f:(X, A) \rightarrow(Y, B)$ is skeletal on the subcomplex $\left(X_{0}, X_{0} \cap A\right)$ of $(X, A)$. Then there exists a skeletal $\varphi$-map $\tilde{f}:(X, A) \rightarrow(Y, B)$ which is $\varphi$-homotopic rel. $\left(X_{0} \cup A\right)$ to $f$.

(In fact the statement in [10] reads ${ }^{2}$ rel. $X_{0^{\prime}}$ but the proof gives the »rel. $\left(X_{0} \cup A\right.$ )» version). Taking $X_{0}=\varnothing$ (or $X_{0}=A$, it amounts to the same) we have the following.

Corollary 1.5. Any $\varphi$-map $f:(X, A) \rightarrow(Y, B)$ is $\varphi$-homotopic rel. $A$ to a skeletal $\varphi$-map $\tilde{f}:(X, A) \rightarrow(Y, B)$. 
Applying Theorem 1.4 to a $\varphi$-map $F:(X, A) \times I \rightarrow(Y, B)$ which is assumed to be skeletal on the subcomplex $(X \times\{0\} \cup X \times\{1\}$, $A \times\{0\} \cup A \times\{1\})$ we get

Collary 1.6. Let $F:(X, A) \times I \rightarrow(Y, B)$ be a $\varphi$-homotopy between the skeletal $\varphi$-maps $f_{0}, f_{1}:(X, A) \rightarrow(Y, B)$. Then there exists a skeletal $\varphi$-homotopy $\tilde{F}:(X, A) \times I \rightarrow(Y, B)$ between $f_{0}$ and $f_{1}$ such that $\tilde{F}|A \times I=F| \mathrm{A} \times I$.

The following facts will be used frequently in this paper without further reference. If $\left(X, X_{0}\right)$ is an equivariant $C W$ pair and $f: X_{0} \rightarrow Y$ is a skeletal $G$-map then the adjuction space $Y \cup_{f} X$ is an equivariant $C W$ complex containing $Y$ as a subcomplex. The mapping cylinder $M_{f}$ of any skeletal $G$-map $f: X \rightarrow Y$ is an equivariant $C W$ complex containing $X$ and $Y$ as subcomplexes ( $X$ in the $\{0\}$ end and $Y$ in the $\{1\}$ end). It follows from Proposition 1.3 that $f$ is a $G$-homotopy equivalence if and only if $X$ is a strong $G$-deformation retract of $M_{f}$. The subcomplex $Y$ is always a strong $G$-deformation retract of $M_{f}$.

Let $\varphi: G \rightarrow G^{\prime}$ be a continuous homorphism. We shall now describe the process of "changing a $G$-space $X$ into a $G^{\prime}$-space, denoted by $\varphi(X)$, through the homomorphism $\varphi$ ». Let $X$ be an arbitrary left $G$-space. Consider the space $G^{\prime} \times X$ and define a right $G$-action $\Phi:\left(G^{\prime} \times X\right) \times$ $G \rightarrow G^{\prime} \times X$ by $\Phi\left(\left(g^{\prime}, x\right), g\right)=\left(g^{\prime} \varphi(g), g^{-1} x\right)$, where $g^{\prime} \in G, g \in G$ and $x \in X$. We define

$$
\varphi(X)=G^{\prime} \times_{\varphi} X
$$

to be the orbit space of $G^{\prime} \times X$ under this right $G$-action. Let $\pi: G^{\prime} \times X \rightarrow \varphi(X)$ be the natural projection and denote $\pi\left(g^{\prime}, x\right)=\left[g^{\prime}, x\right]$. Thus we have $\left[g^{\prime} \varphi(g), x\right]=\left[g^{\prime}, g x\right]$ for every $g \in G$. Now define a left $G^{\prime}$-action

$$
\psi: G^{\prime} \times \varphi(X) \rightarrow \varphi(X)
$$

by $\psi\left(g^{\prime},\left[g_{0}^{\prime}, x\right]\right)=\left[g^{\prime} g_{0}^{\prime}, x\right]$. This completes the construction of the $G^{\prime}$-space $\varphi(X)$. We shall use the notation

$$
\eta: X \rightarrow \varphi(X)
$$

for the canonical $\varphi$-map defined $\eta(x)=[e, x]$, where $e \in G^{\prime}$ is the identity element. Also observe that if $\varphi: G \rightarrow\{e\}=G^{\prime}$ then $\varphi(X)=G \backslash X$, and in this case $\eta: X \rightarrow \varphi(X)$ is the natural projection onto the orbit space.

Any $G$-map $f: X \rightarrow Y$ induces a $G^{\prime}$-map $\varphi(f): \varphi(X) \rightarrow \varphi(Y)$ defined by $\varphi(f)\left(\left[g^{\prime}, x\right]\right)=\left[g^{\prime}, f(x)\right]$. It is immediately seen that $\varphi(f)$ is a welldefined continuous $G^{\prime}$-map. If $h: Y \rightarrow Z$ is another $G$-map we have 
$\varphi(h f)=\varphi(h) \varphi(f)$. It follows that if $f$ is a $G$-homeomorphism then $\varphi(f)$ is a $G^{\prime}$-homeomorphism.

Observe that if $K$ is a topological space with trivial $G$-action then we have $\varphi(X \times K)=\varphi(X) \times K$, as $G^{\prime}$-spaces, given by $\left[g^{\prime}, x, k\right] \rightarrow\left(\left[g^{\prime}, x\right], k\right)$. Thus a $G$-homotopy $F: X \times I \rightarrow Y$ between two $G$-maps $f_{0}$ and $f_{1}$ induces a $G^{\prime}$-homotopy $\varphi(F): \varphi(X) \times I \rightarrow \varphi(Y)$ between the $G^{\prime}$-maps $\varphi\left(f_{0}\right)$ and $\varphi\left(f_{1}\right)$. If $f$ is a $G$-homotopy equivalence then $\varphi(f)$ is a $G^{\prime}$-homotopy equivalence, and if $A$ is a strong $G$-deformation retract of $X$ then $\varphi(A)$ is a strong $G^{\prime}$-deformation retract of $\varphi(X)$.

Also observe that if $Y^{\prime}$ is a $G^{\prime}$-space and $f: X \rightarrow Y^{\prime}$ is a $\varphi$-map then $f$ induces a $G^{\prime}$-map

$$
\varphi(f): \varphi(X) \rightarrow Y^{\prime}
$$

defined by $\varphi(f)\left(\left[g^{\prime}, x\right]\right)=g^{\prime} f(x)$. The fact that we use the same notation $\varphi(f)$ in these two slightly different contexts should not cause any confusion.

Now let $H$ be any closed subgroup of $G$ and consider the $G$-space $G / H$ (standard left $G$-action). We claim that the map

$$
\alpha: \varphi(G / H) \rightarrow G^{\prime} / \varphi(H)
$$

defined by $\alpha\left(\left[g^{\prime}, g H\right]\right)=\left(g^{\prime} \varphi(g)\right) \varphi(H)$ is a $G^{\prime}$-homeomorphism. Since $\alpha\left(\left[g^{\prime} \varphi\left(g_{0}\right), g_{0}^{-1} g H\right]\right)=\left(g^{\prime} \varphi\left(g_{0}\right) \varphi\left(g_{0}\right)^{-1} \varphi(g)\right) \varphi(H)=\left(g^{\prime} \varphi(g)\right) \varphi(H)$ it follows that $\alpha$ is a well-defined continuous map. Clearly $\alpha$ is a $G^{\prime}$-map. The map $\beta: G^{\prime} / \varphi(H) \rightarrow \varphi(G / H)$ defined by $\beta\left(g^{\prime} \varphi(H)\right)=\left[g^{\prime}, e H\right]$ is also immediately seen to be a well-defined continuous $G^{\prime}$-map. Since $\alpha \beta=\mathrm{id}$ and $\beta \alpha=\mathrm{id}$ this shows that $\alpha$ is a $G^{\prime}$-homeomorphism. We identify $\varphi(G / H)$ with $G^{\prime} / \varphi(H)$ through $\alpha$. It follows that we have

$$
\varphi\left(G / H \times I^{n}\right)=G^{\prime} / \varphi(H) \times I^{n}
$$

Using this fact one easily proves the following

Proposition 1.7. If $(X, A)$ is a $G$-equivariant relative $C W$ complex then $(\varphi(X), \varphi(A))$ is a $G^{\prime}$-equivariant relative $C W$ complex.

If $f:(X, A) \rightarrow(Y, B)$ is skeletal then also $\varphi(f):(\varphi(X), \varphi(A)) \rightarrow$ $(\varphi(Y), \varphi(B))$ is skeletal.

\section{Chapter II. Foundations of equivariant simple-homotopy theory}

Recall that $G$ denotes a topological group which is either a compact Lie group or a discrete group. By »equivariant» we mean "G-equivariant» if not otherwise is specified. Only when two groups $G$ and $G^{\prime}$ are involved in the discussion at the same time shall we be more specific and speak 
about $G$-equivariant and $G^{\prime}$-equivariant. From now on all equivariant $C W$ complexes are automatically assumed to be finite equivariant $C W$ complexes. Thus we shall write "equivariant $C W$ complex (pair)» when we in fact mean »finite equivariant $C W$ complex (pair)».

\section{§ 1. Equivariant formal deformations}

Definition 1.1. An inclusion $i: X \rightarrow Y$ of equivariant $C W$ complexes is called an equivariant elementary expansion if the equivariant $C W$ pair $(Y, i(X))$ satisfies the following conditions.

1) There is an integer $n \geq 1$ such that

$$
Y=i(X) \cup b^{n-1} \cup b^{n},
$$

where $b^{n-1}$ and $b^{n}$ denote an open equirariant $(n-1)$-cell and an open equivariant $n$-cell, respectively, of $Y-i(X)$.

2) There exists a closed subgroup $H$ of $G$ and a $G$-map

$$
\sigma: G / H \times I^{n} \rightarrow Y
$$

such that

(b) $\sigma: G / H \times I^{n-1} \rightarrow Y$ is a characteristic $G$-map for the equirariant $(n-1)$-cell $\bar{b}^{n-1}$.

(c) $\sigma$ is a characteristic $G$-map for the equivariant $n$-cell $\bar{b}^{n}$.

By definition it follows that if $i: X \rightarrow Y$ is an equivariant elementary expansion and $h: X^{\prime} \cong X$ is an isomorphism of equivariant $C W$ complexes then also ih: $\vec{X}^{\prime} \rightarrow Y$ is an equivariant elementary expansion. Since we also define the identity map $i d: X \rightarrow X$ to be an equivariant elementary expansion it follows that any isomorphism $h: X^{\prime} \cong X$ of equivariant $C W$ complexes is an equirariant elementary expansion. It is also immediately seen that if $i: X \rightarrow Y$ is an equirariant elementary expansion and $\bar{h}: Y \cong Y^{\prime}$ is an isomorphism of equirariant $C W$ complexes then $\bar{h} i: X \rightarrow \vec{Y}^{\prime}$ is an equirariant elementary expansion.

We shall in the following identify $X$ with $i(X)$ and consider $X$ itself as a subcomplex of $Y$. Hence we also use the terminology $" Y$ is an equivariant elementary expansion of $X_{\text {》 }}$ and denote $Y=X \cup b^{n-1} \cup b^{n}$. Observe that the open equivariant cells $b^{n-1}$ and $b^{n}$ have the same type and we call this for the type of the equivariant elementary expansion and the integer $n$ for its dimension. Any $G$-map $\sigma: G / H \times I^{n} \rightarrow Y$ which 
satisfies the conditions in Definition 1.1 will be called a characteristic simple $G$-map for $\left(b^{n}, b^{n-1}\right)$.

The conditions $(a)-(c)$ in Definition 1.1 are equivalent to the four conditions that, $\sigma\left(G / H \times J^{n-1}\right) \subset X^{n-1}, \sigma\left(G / H \times \partial I^{n-1}\right) \subset X^{n-2}, \sigma$ maps $G / H \times\left(I^{n-1}-\partial I^{n-1}\right) \quad G$-homeomorphically onto $b^{n-1}$, and $\sigma$ maps $G / H \times\left(I^{n}-\partial I^{n}\right) \quad G$-homeomorphically onto $b^{n}$. Thus $Y$ is an equivariant elementary expansion of $X$ if and only if $Y$ is the adjunction space of $G / H \times I^{n}$, for some closed subgroup $H$ of $G$, and $X$ by a $G$-map $\varphi_{+}: G / H \times J^{n-1} \rightarrow X^{n-1}$ which also satisfies $\varphi_{\div}\left(G / H \times \partial I^{n-1}\right) \subset X^{n-2}$.

We use the terminology $\gg$ is an equivariant elementary collapse of $Y_{\text {» }}$ to mean exactly the same thing as $» Y$ is an equivariant elementary expansion of $X »$. Observe that a strong $G$-deformation retraction $\tilde{F}:\left(G / H \times I^{n}\right) \times I \rightarrow G / H \times I^{n}$ of $G / H \times I^{n}$ to $G / H \times J^{n-1}$ and a characteristic simple $G$-map $\sigma: G / H \times I^{n} \rightarrow Y$ for $\left(b^{n}, b^{n-1}\right)$ together give rise to a strong $G$-deformation retraction $F: Y \times I \rightarrow Y$ of $Y$ to $X$. Let $r: Y \rightarrow X$ denote the corresponding $G$-retraction. Thus the inclusion $i: X \rightarrow Y$ is a $G$-homotopy equivalence and $r$ is a $G$-homotopy inverse to $i$. In fact any $G$-retraction from $Y$ onto $X$ is a $G$-homotopy inverse to the inclusion $i: X \rightarrow Y$ and any two such $G$-retractions are $G$-homotopic rel. $X$. Thus regardless of the different choices of $\tilde{F}$ and $\sigma$ the $G$-retraction $r: Y \rightarrow X$ is uniquely determined up to $G$-homotopy rel. $X$. We call $r: Y \rightarrow X$ for an equivariant elementary collapse. A $G$-map which is either an equivariant elementary expansion or collapse is called an equivariant elementary deformation.

Let $(V, X)$ and $(W, X)$ be two equivariant $C W$ pairs. We define an equivariant formal deformation from $V$ to $W$ rel. $X$ to be a finite composite $k=k_{p} \ldots k_{1}$ of equivariant elementary deformations $k_{i j}$,

$$
V=X_{0} \stackrel{k_{1}}{\rightarrow} X_{1} \stackrel{k_{2}}{\rightarrow} \cdots \stackrel{k_{p}}{\rightarrow} X_{p}=H^{r}
$$

where each $X_{j}$ contains $X$ as a subcomplex and $k_{j} \mid X=i d, j=1, \ldots, p$. (Here $k_{j} \mid X=i d$ means that; if $i_{j}: X \rightarrow X_{j}$ is the inclusion representing $X$ as a subcomplex of $X_{j}$ then $k_{j} i_{j-1}=i_{j}$, for $j=1, \ldots, p$.) Let $k_{j}: X_{j} \rightarrow X_{j-1}$ be the equivariant elementary deformation inverse to $k_{j}$. Then $\bar{k}=\bar{k}_{1} \ldots \bar{k}_{p}$ is a G-homotopy inverse, rel $X$, to $k$, and $\bar{k}$ is an equivariant formal deformation from $W$ to $V$ rel. $X$. We say that $V$ and $W$ have the same equivariant simple-homotopy type rel. $X$ if and only if there exists an equivariant formal deformation from $V$ to $W$ rel. $X$. We denote this by

$$
V \text { s } W \text { rel. } X \text {, }
$$

adding the word »equivariantly» when we want to be very specific. If each 
$k_{j}: X_{j-1} \rightarrow X_{j}$ is an equivariant elementary collapse (expansion) we say that $k=k_{p} \ldots k_{1}$ is an equivariant collapse (expansion) and we also express this by saying that $V$ collapses (expands) equivariantly to $W$. Observe that in these special cases we in particular have $V \mathrm{~s} W$ rel. $W$ (and rel. $V$, respectively).

We define a $G$-map $f: V \rightarrow W$, where $f \mid X=\mathrm{id}$, to be an equivariant simple-homotopy equivalence rel. $X$ if and only if $f$ is $G$-homotopic rel. $X$ to an equivariant formal deformation $k: V \rightarrow W$, which thus also is rel. $X$.

If $G=\{e\}$, the trivial group, these definitions reduce to the corresponding definitions in the ordinary "non-equivariant» case, see Section 13 in Whitehead [18].

An equivariant simple-homotopy equivalence $f: X \rightarrow Y$ induces an ordinary simple-homotopy equivalence $f^{\prime}: G \backslash X \rightarrow G \backslash Y$ on the orbit spaces. But $f$ need not be an equivariant simple-homotopy equivalence even if the induced map on the orbit spaces is an ordinary simple-homotopy equivalence. Consider the following simple example. Let $Y$ be the twosphere $S^{2}$ with $G=S^{1}$ acting by the standard "free» rotation leaving the south pole $\{S\}$ and the north pole $\{N\}$ fixed. The orbit space is a unit interval which collapses to $\{0\}$. But the inclusion $i:\{S\} \rightarrow Y$ is not an equivariant simple-homotopy equivalence. Of course in this example the $G$-map $i$ is not even a $G$-homotopy equivalence. We shall give a better example later on.

Lemma 1.2. Let $(V, X)$ be an equivariant $C W$ pair such that $V$ s $X$ rel. $X$. Then both the inclusion $i: X \rightarrow V$ and any $G$-retraction $r: V \rightarrow X$ are equivariant simple-homotopy equivalences.

Proof. Since $X$ s $V$ rel. $X$ the inclusion $i: X \rightarrow V$ is an equivariant formal deformation and hence an equivariant simple-homotopy equivalence. Since $X$ is a strong $G$-deformation retract of $V$ it follows that any $G$ retraction $r: V \rightarrow X$ is a $G$-homotopy inverse to $i$ and hence also an equivariant simple-homotopy equivalence.

Lemma 1.3. Let $f: X \rightarrow Y$ be an equivariant simple-homotopy equivalence, and let $K$ be any closed subgroup of $G$. Then $f: G X^{K} \rightarrow G Y^{K}$ is an equivariant simple-homotopy equivalence.

Proof. Let $B=A \cup b^{n-1} \cup b^{n}$ be an equivariant elementary expansion of $A$, of say type $(H)$. Then $G B^{K}=G A^{K} \cup b^{n-1} \cup b^{n}$ if $(K) \leq(H)$ and $G B^{K}=G A^{K}$ if $(K) \$(H)$, that is, $G B^{K}$ is in either case an equivariant elementry expansion of $G A^{K}$. It follows that if $k: X \rightarrow Y$ is an equivariant formal deformation then so is also $k \mid: G X^{K} \rightarrow G Y^{K}$. Since any $G$-homotopy from $f$ to $k$ restricts to a G-homotopy from $f \mid$ to $k \mid$ this 
shows that $f \mid: G X^{K} \rightarrow G Y^{K}$ is an equivariant simple-homotopy equivalence.

Lemma 1.4. Let $Y$ be a $G$-equivariant elementary expansion of $X$, and let $\varphi: G \rightarrow G^{\prime}$ be a continuous homomorphism. Then $\varphi(Y)$ is a $G^{\prime}$ equivariant elementary expansion of $\varphi(X)$.

Proof. Denote $Y=X \cup b^{n-1} \cup b^{n}$, and assume that the type of the equivariant elementary expansion is $(H)$. Let $\sigma: G / H \times I^{n} \rightarrow Y$ be a characteristic simple $G$-map for $\left(b^{n}, b^{n-1}\right)$. We have $\varphi(Y)=$ $\varphi(X) \cup \varphi\left(b^{n-1}\right) \cup \varphi\left(b^{n}\right)$, and since $\varphi\left(G / H \times I^{n}\right)=G^{\prime} / \varphi(H) \times I^{n}$ it is easily seen that $\varphi(\sigma): G^{\prime} / \varphi(H) \times I^{n} \rightarrow \varphi(Y)$ is a characteristic simple $G^{\prime}$-map for $\left(\varphi\left(b^{n}\right), \varphi\left(b^{n-1}\right)\right)$. Thus $\varphi(Y)$ is a $G^{\prime}$-equivariant elementary expansion, of type $\varphi(H)$, of $\varphi(X)$.

Corollary 1.5. Let $(V, X)$ and $(W, X)$ be $G$-equivariant $C W$ pairs such that $V$ s $W$ rel. $X \quad G$-equivariantly. Then we have $\varphi(V) \mathrm{s} \varphi(W)$ rəl. $\varphi(X) G^{\prime}$-equivariantly.

Both Lemma 1.6 and Corollary 1.7 below will be used frequently in the following. We shall call both of them for the "relativity principle».

Lemma 1.6. Assume that $(V, X)$ and $(W, X)$ are equivariant CW pairs such that $V$ s $W$ rel. $X$. Let $f: X \rightarrow Y$ be a skeletal $G$-map. Then $\left(Y \mathrm{\cup}_{f} V\right) \mathrm{s}\left(Y \mathrm{\cup}_{f} W\right)$ rel. $Y$.

Proof. Let $V=X_{0} \rightarrow X_{1} \rightarrow \ldots \rightarrow X_{p}=W$ be an equivariant formal deformation rel. $X$. Denote $Y_{i}=Y \cup_{f} X_{i}, i=0, \ldots, p$. It is then immediately seen that $Y \cup_{f} V=Y_{0} \rightarrow Y_{1} \rightarrow \ldots \rightarrow Y_{p}=Y \cup_{f} W$ is an equivariant formal deformation rel. $Y$.

Observe in particular the special case of Lemma 1.6. when $f$ is an inclusion. By Corollary 1.5 and Lemma 1.6 we have

Corollary 1.7. Let $(V, X)$ and $(W, X)$ be as in Corollary 1.5. and let $f: X \rightarrow Y^{\prime}$ be a skeletal $\varphi$-map. Then we have $\left(Y^{\prime} \cup_{\varphi(f)} \varphi(W)\right) \mathrm{s}\left(Y^{\prime}\right.$ $\left.\mathrm{U}_{\varphi(f)} \varphi(V)\right)$ rel. $Y^{\prime} G^{\prime}$-equivariantly.

The following lemma and its two corollaries will be used frequently. The "same" lemma in the ordinary non-equivariant case is Lemma 11 in Whitehead [18].

Lemma 1.8. Let $f: X \rightarrow Y$ be a skeletal $G$-map and let $X_{0}$ be a subcomplex of $X$. Then $M_{f}$ collapses equivariantly to $M_{f \mid X_{0}}$.

Proof. Let A be a subcomplex of $X$ such that $X=A \cup b$, where $b$ is an open equivariant, say, $n$-cell of $X$. We claim that $M_{f \mid A}$ is an equivariant elementary collapse of $M_{f}$. Assume that the type of $b$ is $(H)$ 
and let $\alpha: G / H \times I^{n} \rightarrow X$ be a characteristic $G$-map for $\bar{b}$. We have $M_{f}=M_{f \mid A} \cup(b \times\{0\}) \cup(b \times(0,1)) \quad$ and $\pi(\alpha \times$ id $): G / H \times I^{n} \times$ $I \rightarrow M_{f}$, where $\pi: X \times I \rightarrow M^{n}$ denotes the restriction of the natural projection, is clearly a characteristic simple $G$-map for $(b \times(0,1), b \times\{0\})$. This proves the above claim. Now let $X_{0} \subset X_{1} \subset \ldots \subset X_{m}=X$ be subcomplexes of $X$ such that $X_{i}-X_{i-1}$ consists of exactly one open equivariant cell, for $i=1, \ldots, m$. By what we just showed $M_{f \mid X_{i-1}}$ is an equivariant elementary collapse of $M_{f \mid x_{i}}, i=1, \ldots, m$. This completes the proof of the lemma.

Corollary 1.9. Let $f: X \rightarrow Y$ be a skeletal $G$-map. Then $M_{f}$ collapses equivariantly to $Y$.

Corollary 1.10. Let $\left(X, X_{0}\right)$ be an equivariant $C W$ pair. Then $X \times I$ collapses equivariantly to $X_{0} \times I \cup X \times\{1\}$, and hence of course also to $X_{0} \times I \cup X \times\{0\}$.

\section{§ 2. The equivariant Whitehead group $\mathrm{Wh}_{\mathrm{G}}(\mathrm{X})$}

Let $(V, X)$ be an equivariant $C W$ pair such that $X$ is a strong $G$ deformation retract of $I$. (By Proposition 1.3 in Chapter $I$ this is equivalent to the fact that the inclusion $i: X \rightarrow V$ is a $G$-homotopy equivalence.) Let $(W, X)$ be another such pair. Define a relation $\sim$ by

$$
(V, X) \sim(W, X) \Leftrightarrow V \text { s } W \text { rel. } X \text { equivariantly . }
$$

This is an equivalence relation. Since $(V, X) \sim(W, X)$ if $(V, X) \cong$ $(W, X)$, where $\cong$ stands for an isomorphism of equivariant $C W$ complexes which is the identity on $X$, it is easy to see that the equivalence classes with respect to the relation $\sim$ form a set. We denote this set by $W h_{G}(X)$. Let $s(V, X)$ denote the equivalence class determined by $(V, X)$. Now define an addition in $\mathbb{W} h_{G}(X)$ by

$$
s\left(V_{1}, X\right) \doteq s\left(\Gamma_{2}, X\right)=s\left(\Gamma_{1} U_{X} \Gamma_{2}, X\right) .
$$

Since $X$ is a strong $G$-deformation retract of both $V_{1}$ and $V_{2}$ it follows that $X$ is a strong $G$-deformation retract of $r_{1} \cup_{X} V_{2}$. Thus $s\left(V_{1} \cup_{X} V_{2}, X\right) \in W h_{G}(X)$ is defined. This addition is well-defined. If $\left(V_{1}, X\right) \sim\left(W_{1}, X\right)$ and $\left(V_{2}, X\right) \sim\left(H_{2}, X\right)$ then it follows from the relativity principle, Proposition 1.6, that we have $\left(V_{1} \cup_{X} V_{2}, X\right) \sim$ $\left(V_{1} \cup_{X} W_{2}, X\right) \sim\left(W_{1} \cup_{X} W_{2}, X\right)$. Clearly this addition is associative and commutative and the element $s(X, X) \in W h_{G}(X)$ is a zero element. We shall shortly show that every element in $W h_{G}(X)$ has an inverse, i.e., $W h_{G}(X)$ is an abelian group. But first we establish some other results. 
Let $f: X \rightarrow Y^{\prime}$ be a skeletal $\varphi$-map, where $\varphi: G \rightarrow G^{\prime}$ is a continuous homomorphism and $Y^{\prime}$ is a $G^{\prime}$-equivariant $C W$ complex. We define

$$
f_{*}: W h_{G}(X) \rightarrow W h_{G^{\prime}}\left(Y^{\prime}\right)
$$

as follows. If $s(V, X) \in W h_{G}(X)$ then we set

$$
f_{*} s(V, X)=s\left(Y^{\prime} \cup_{\varphi(f)} \varphi(V), Y^{\prime}\right),
$$

where $\varphi(f): \varphi(X) \rightarrow Y^{\prime}$ denotes the $G^{\prime}$-map induced by the $\varphi$-map $f: X \rightarrow Y^{\prime}$. It is easily seen that this definition makes sense and it follows from the relativity principle, Corollary 1.7, that it is well-defined. Clearly $f_{*}$ is additive and takes the zero element into the zero element, i.e., $f_{*}$ is a homomorphism between abelian semi-groups with zero element. If $h: Y^{\prime} \rightarrow Z^{\prime \prime}$ is a $\varphi^{\prime}$-map, where $\varphi^{\prime}: G^{\prime} \rightarrow G^{\prime \prime}$ is a continuous homomorphism, then we have $(h f)_{*}=h_{*} f_{*}: W h_{G}(X) \rightarrow W h_{G^{\prime \prime}}\left(Z^{\prime \prime}\right)$. In particular the canonical $\varphi$-map $\eta: X \rightarrow \varphi(X)$ induces a homomorphism which we shall denote by $\varphi_{*}$ instead of $\eta_{*}$. Thus we have

$$
\varphi_{*}: W h_{G}(X) \rightarrow W h_{G^{\prime}}(\varphi(X)),
$$

for any continuous homomorphism $\varphi: G \rightarrow G^{\prime}$, and $\varphi_{*}$ is defined by $\varphi_{*} s(V, X)=s(\varphi(V), \varphi(X))$.

Lemma 2.1. Let $f_{0}, f_{1}: X \rightarrow I^{\prime}$ be skeletal $\varphi$-maps which are $\varphi^{-}$ homotopic. Then $\left(f_{0}\right)_{*}=\left(f_{1}\right)_{*}: W h_{G}(X) \rightarrow W h_{G^{\prime}}\left(Y^{\prime}\right)$.

Proof. Let $F: X \times I \rightarrow Y$ be a $\varphi$-homotopy from $f_{0}$ to $f_{1}$. By the equivariant skeletal approximation theorem (Corollary 1.6 in Chapter I) we can assume that $F$ is skeletal. Thus $F$ induces

$$
F_{*}: W h_{G}(X \times I) \rightarrow W h_{G^{\prime}}\left(Y^{\prime}\right) .
$$

Now consider the inclusions $i_{0}, i_{1}: X \rightarrow X \times I$, defined by $i_{k}=$ $(x, k), k=0,1$. Let $s(V, X) \in W h_{G}(X)$. Using Corollary 1.10 we then have $\left(i_{0}\right)_{*} s(V, X)=s(X \times I \cup V \times\{0\}, X \times I)=s(V \times I, X \times I)=$ $\mathrm{s}(X \times I \cup V \times\{1\}, X \times I)=\left(i_{1}\right)_{*} s(V, X)$. Thus $\left(i_{0}\right)_{*}=\left(i_{1}\right)_{*}: W h_{G}(X)$ $\rightarrow W h_{G}(X \times I)$. Hence we have $\left(f_{0}\right)_{*}=F_{*}\left(i_{0}\right)_{*}=F_{*}\left(i_{1}\right)_{*}=\left(f_{1}\right)_{*}$.

Lemma 2.2. Let $X \subset V \subset W$, such that $X$ is a strong $G$-deformation retract of $V$ and $V$ is a strong $G$-deformation retract of $W$. Then we have

$$
s(W, X)=r_{*} s(W, V)+s(V, X),
$$

where $r: V \rightarrow X$ is a skeletal G-retraction.

Proof. First we observe that it follows that $X$ is a strong $G$-deformation retract of $W$ and hence $s(W, X) \in W h_{G}(X)$ is defined. Let $i: X \rightarrow V$ be the inclusion and $r: V \rightarrow X$ a skeletal $G$-retraction. Then ir $V \rightarrow V$ 
is $G$-homotopic (in fact rel. $X$ ) to $i d_{V}$. Thus by Lemma 2.1 we have $(i r)_{*} s(W, V)=s(W, V)$. This means that $V \cup_{i r} W \mathrm{~s} W$ rel. $V$ and hence in particular rel. $X$. Thus $s\left(V \cup_{i r} W, X\right)=s(W, X)$ and since $s\left(V \cup_{i r} W, X\right)=s(V, X)+s\left(X \cup_{r} W, X\right)=s(V, X)+r_{*} s(W, V)$ the lemma follows.

We are now ready to prove that every element in $W h_{G}(X)$ has an inverse. Let $s(V, X) \in W h_{G}(X)$ and let $r: V \rightarrow X$ be a skeletal $G$ retraction. By Lemma 1.8. the mapping cylinder $M_{r}$ collapses equivariantly to $X \times I$, and thus $M_{r} \mathrm{~s} X \times I$ rel. $X \times I$. Let $\pi: X \times I \rightarrow X$ denote the projection and define $\bar{M}_{r}=X \cup_{\pi} M_{r}$. By the relativity principle, Lemma 1.6, we have $\bar{M}_{r} \mathrm{~s} X$ rel. $X$, and hence $s\left(\bar{M}_{r}, X\right)=0 \in W h_{G}(X)$. Now $X \subset V \subset \bar{M}_{r}$, and since $X \times I \cup V \times\{0\}$ is a strong $G$-deformation retract of $M_{r}$ it follows that $V$ is a strong $G$-deformation retract of $\bar{M}_{r}$. Thus Lemma 2.2 applies and gives us

$$
0=r_{*} s\left(\bar{M}_{r}, V\right)+s(V, X),
$$

that is, $r_{*} s\left(\bar{\Pi}_{r}, V\right) \in W h_{G}(X)$ is an inverse to $s(V, X)$. We have proved

Theorem 2.3. For every G-equivariant $C W$ complex $X W h_{G}(X)$ is an abelian group. A $\varphi$-map $f: X \rightarrow Y^{\prime}$ induces a homomorphism $f_{*}: W h_{G}(X) \rightarrow W h_{G^{\prime}}\left(Y^{\prime}\right)$ and any two $\varphi$-homotopic $\varphi$-maps induce the same homomorphism.

We call $W h_{G}(X)$ for the equivariant Whitehead group of $X$. If $G=\{e\}$, the trivial group, and $X$ hence denotes an ordinary $C W$ complex it is clear from the way we have defined our $W h_{G}(X)$ that $W h_{\{e\}}(X)=$ $W h(X)$. Here $W h(X)$ is the Whitehead group of the $C W$ complex $X$ as defined in Cohen [6], Eckmann-Maumary [7], and Stöcker [15]. It is also well-known that this ordinary Whitehead group $W h(\mathrm{X})$, for comnected $X$, is isomorphic to $W h\left(\tau_{1}(X)\right)$, the (algebraicly defined) Whitehead group of the group $\tau_{1}(X)$. For the proof of this see Stöcker [15] or Cohen [6].

The following type of "sum theorem» has played an important role in the ordinary simple-homotopy theory. The proof of our equivariant version of the sum theorem is exactly the same" as the one given by Stöcker [15] for the ordinary case.

Proposition 2.4. Let $(H, X)$ be an equirariant $C W$ pair such that $W=W_{1} \cup W_{2}$ and $W_{0}=W_{1} \cap W_{2}$. Denote $X_{k}=X \cap W_{k} \quad$ and assume that $X_{k}$ is a strong $G$-deformation retract of $W_{k}, k=0,1,2$. Then we have

$$
s(W, X)=\left(i_{1}\right)_{*} s\left(W_{1}, X_{1}\right)+\left(i_{2}\right)_{*} s\left(W_{2}, X_{2}\right)-\left(i_{0}\right)_{*}\left(W_{0}, X_{0}\right),
$$

where $i_{k}: X_{k} \rightarrow X, k=0,1,2$, denote the inclusions. 
Proof. We have $X=X_{1} \cup X_{2}$ and $X_{0}=X_{1} \cap X_{2}$. Denote $V_{k}=$ $X \cup W_{k}, k=0,1,2$. Clearly $X$ is a strong $G$-deformation retraction of $V_{k}$, for $k=0,1,2$. Since $X \subset V_{0} \subset V_{j}, j=1,2$, it follows that also the inclusions $V_{0} \rightarrow V_{j}, j=1,2$, are $G$-homotopy equivalences and hence by Proposition 1.3 in Chapter $\mathrm{I} V_{0}$ is a strong $G$-deformation retract of $V_{j}, j=1,2$. Since $W=V_{1} \cup V_{2}$ and $V_{1} \cap V_{2}=V_{0}$ we have

$$
s\left(W, V_{0}\right)=s\left(V_{1}, V_{0}\right)+s\left(V_{2}, V_{0}\right)
$$

by the definition of the sum in $W h_{G}\left(V_{0}\right)$. Let $r: V_{0} \rightarrow X$ be a retraction. Then we have by Lemma 2.2

$$
\begin{aligned}
& s(W, X)=r_{*} s\left(W, V_{0}\right)+s\left(V_{0}, X\right) \\
& s\left(V_{j}, X\right)=r_{*} s\left(V_{j}, V_{0}\right)+s\left(V_{0}, X\right), j=1,2 .
\end{aligned}
$$

From the above four formulas we get

$$
s(W, X)-s\left(V_{1}, X\right)-s\left(V_{2}, X\right)=-s\left(V_{0}, X\right)
$$

and this is the claimed formula since $\left(i_{k}\right)_{*} s\left(W_{k}, X_{k}\right)=s\left(V_{k}, X\right), k_{k}=$ $0,1,2$.

We shall now study the case when $G$ acts freely on $X$ and prove that $\varphi_{*}: W h_{G}(X) \rightarrow W h(G \backslash X)$ is an isomorphism. Recall that if $\varphi: G \rightarrow\{e\}$ then $\varphi(X)=G \backslash X$ and hence this $\varphi$ gives us $\varphi_{*}: W h_{G}(X) \rightarrow W h(G \backslash X)$. We first give two lemmas.

Lemma 2.5. Let $(Y, X)$ be an equivariant $C W$ pair such that $G$ acts freely on $Y$. Assume that $G \backslash X$ is an elementary collapse (in the ordinary sense) of $G \backslash Y$. Then $X$ is an equivariant elementary collapse of $Y$.

Proof. Let us denote $\left(Y^{\prime}, X^{\prime}\right)=(G \backslash Y, G \backslash X)$ and let $p: Y \rightarrow Y^{\prime}$ denote the projection onto the orbit space. By the assumption we have $Y^{\prime}=X^{\prime} \cup e^{n-1} \cup e^{n}$, where $e^{n-1}$ and $e^{n}$ denote an open $(n-1)$-cell and $n$-cell, respectively, of $Y^{\prime}-X^{\prime}$. Moreover there is a map $\sigma^{\prime}: I^{n} \rightarrow Y^{\prime}$ such that $\sigma^{\prime}\left(J^{n-1}\right) \subset\left(X^{\prime}\right)^{n-1}$ and $\sigma^{\prime}\left(\partial I^{n-1}\right) \subset\left(X^{\prime}\right)^{n-2}$, and furthermore $\sigma^{\prime}$ maps $I^{n-1}-\partial I^{n-1}$ homeomorphically onto $e^{n-1}$ and $I^{n}-\partial I^{n}$ homeomorphically onto $e^{n}$. Since $p: Y \rightarrow Y^{\prime}$ is a principal $G$-bundle and $I^{n}$ is contractible it follows that $\sigma^{\prime}$ can be lifted, that is, there exists a map $\tilde{\sigma}: I^{n} \rightarrow Y$ such that $p \tilde{\sigma}=\sigma^{\prime}$. Now define $\sigma: G \times I^{n} \rightarrow Y$ by $\sigma(g, x)=$ $g \tilde{\sigma}(x)$. Then $\sigma$ is a $G$-map such that $\sigma\left(G \times J^{n-1}\right) \subset X^{n-1}$ and $\sigma\left(G \times \partial I^{n-1}\right)$ $\subset X^{n-2}$, and moreover $\sigma$ maps $G \times\left(I^{n-1}-\partial I^{n-1}\right) G$-homeomorphically onto $p^{-1}\left(e^{n-1}\right)$ and $G \times\left(I^{n}-\partial I^{n}\right) \quad G$-homeomorphically onto $p^{-1}\left(e^{n}\right)$. 
Since $Y=X \cup p^{-1}\left(e^{n-1}\right) \cup p^{-1}\left(e^{n}\right)$ this shows that $X$ is an equivariant elementary collapse of $Y$.

Lemma 2.6. Let $X$ be an equivariant $C W$ complex such that $G$ acts freely on $X$. Let $Y^{\prime}$ be an elementary expansion in the ordinary sense of $G \backslash X$. Then there exists an equivariant elementary expansion $Y$ of $X$ such that $G \backslash Y=Y^{\prime}$.

Proof. Denote $X^{\prime}=G \backslash X$. By the assumption $Y^{\prime}$ is the adjunction space of $X^{\prime}$ and $I^{n}$ by some map $\varphi_{+}^{\prime}: J^{n-1} \rightarrow\left(X^{\prime}\right)^{n-1}$ which also satisfies $q_{+}^{\prime}\left(\partial I^{n-1}\right) \subset\left(X^{\prime}\right)^{n-2}$, that is, $Y^{\prime}=X^{\prime} \cup_{q_{+}^{\prime}} I^{n}$. Since $p: X \rightarrow X^{\prime}$ is a principal $G$-bundle and $J^{n-1}$ is contractible there exists a lifting $\tilde{\sigma}_{+}: I^{n}$ $\rightarrow X^{n-1} \subset X$ of $\sigma_{+}^{\prime}$. Let $\sigma_{+}: G \times I^{n} \rightarrow X^{n-1}$ be defined by $\sigma_{+}(g, a)=$ $g \sigma_{+}(a)$. Then we also have $\sigma_{+}\left(G \times \partial I^{n-1}\right) \subset X^{n-2}$. Thus $Y=X \mathrm{U}_{\varphi_{+}}\left(G \times I^{n}\right)$ satisfies the conclusion of the lemma.

Theorem 2.7. Let $X$ be an equirariant $C H$ complex such that $G$ acts freely on $X$. Then $\mathscr{f}_{*}: W h_{G}(X) \rightarrow W h(G \backslash X)$ is an isomorphism.

Proof. Let $s(V, X) \in W h_{G}(X)$ and assume that $\varphi_{*} s(V, X)=$ $(G \backslash V, G \backslash X)=0 \in W h(G \backslash X)$. This means that $G \backslash V$ s $G \backslash X$ rel. $G \backslash X$. By Lemma 2.5 and 2.6 it follows that $V \mathrm{~s} X$ rel. $X$ equivariantly, that is, $s(V, X)=0$. Thus $\varphi_{*}$ is a monomorphism. Now let $s\left(V^{\prime}, G \backslash X\right) \in$ $W h(G \backslash X)$. Let $r^{*}(p): V \rightarrow V^{\prime}$ be the principal $G$-bundle induced from $p: X \rightarrow G \backslash X$ by a retraction $r: V^{\prime} \rightarrow G \backslash X$. Then $V$ is an equivariant $C W$ complex on which $G$ acts freely and $V$ contains $X$ as a subcomplex. Since the inclusion $\tilde{i}: X \rightarrow V$ induces isomorphisms on all homotopy groups it follows by Corollary 5.5 in Bredon [4] in case $G$ is a discrete group and by Proposition 2.5 in [9] in case $G$ is a compact Lie group, that $\tilde{i}$ is a $G$-homotopy equiralence. Thus, br Proposition 1.3 in Chapter I , $X$ is a strong $G$-deformation retract of $V$, and the equivariant $C W$ pair $(V, X)$ determines an element $s(T, X) \in W h_{G}(X)$. Since $\varphi_{*} s(V, X)=$ $\left(V^{\prime}, G \backslash X\right)$ this shows that $\mathscr{\varphi}_{*}$ is an epimorphism.

Let $G / G_{0}$ be the group of components of $G$, i.e. $G_{0}$ denotes the identity component of $G$. We now have

Corollary 2.8. Let $X$ be as in Theorem 2.7 and assume moreover that $X$ is simply connected. Then $W h_{G}(X) \cong W h\left(G / G_{0}\right)$.

Proof. It follows from the exact homotopy sequence of the fibration $p: X \rightarrow G \backslash X$ that $\pi_{1}(G \backslash X)=G / G_{0}$. Hence the corollary follows from Theorem 2.7 and the well-known isomorphism $W h(G \backslash X) \cong W h\left(\pi_{1}(G \backslash X)\right)$, see Section 3 in Stöcker [15] or $§ 21$ in Cohen [6]. 


\section{§ 3. Geometric equivariant Whitehead torsion of a G-homotopy equivalence}

In this section we first give a characterization of an equivariant simplehomotopy equivalence in terms of its mapping cylinder, see Theorem 3.6 below. The proofs of this result and of those preceeding it are completely analogous to the ones in the ordinary "non-equivariant» case, see (5.4) (5.8) in Cohen [6] or $\S 3$ in Eckmann-Maumary [7]. Then we define the (geometric) equivariant Whitehead torsion of a $G$-homotopy equivalence $f: X \rightarrow Y$. It is an element of $W h_{G}(X)$, (see the remarks below concerning this choice). We denote it by $\tau_{g}(f)$, and we have $\tau_{g}(f)=0$ if and only if $f$ is an equivariant simple-homotopy equivalence. Recently Chapman [5] has given an affirmative answer to the outstanding problem concerning the topological invariance of ordinary Whitehead torsion for finite $C W$ complexes. From this result it follows immediately, by Theorem 2.7, that if $X$ and $Y$ are equivariant $C W$ complexes such that $G$-acts freely then any $G$-homeomorphism $f: X \rightarrow Y$ is an equivariant simple-homotopy equivalence.

Lemma 3.1. Assume that $V$ collapses equivariantly to $X$. Let $f: V \rightarrow Y$ be a skeletal $G$-map. Then $M_{f}$ collapses equivariantly to $V \cup M_{f \mid X}$, where $f \mid X: X \rightarrow Y$.

Proof. Let $V=X_{m} \rightarrow X_{m-1} \rightarrow \ldots \rightarrow X_{0}=X$ be a sequence of equivariant elementary collapses. Denote $W_{j}=V \cup M_{f \mid x_{j}}$. We claim that $W_{j-1}$ is an equivariant elementary collapse of $W_{j}, 1 \leq j \leq m$. This is seen as follows. Assume that the equivariant elementary collapse from $X_{j}$ to $X_{j-1}$ is of type $\left(H_{j}\right)$, and denote $X_{j}=X_{j-1} \cup b^{n-1} \cup b^{n}$, and let $\sigma: G / H_{j} \times I^{n} \rightarrow X_{j}$ be a characteristic simple $G$-map for $\left(b^{n}, b^{n-1}\right)$. Then we have $W_{j}=W_{j-1} \cup\left(b^{n-1} \times(0,1)\right) \cup\left(b^{n} \times(0,1)\right)$ and $\pi(\sigma \times i d)$ : $G / H_{j} \times I^{n} \times I \rightarrow W_{j}$, where $\pi: X_{j} \times I \rightarrow W_{j}$ denotes the restriction of the natural projection, is clearly a characteristic simple $G$-map for $\left(b^{n} \times(0,1), b^{n-1} \times(0,1)\right)$. (The $G$-map $\pi(\sigma \times i d)$ restricted to $G / H_{j} \times\left(I^{n-1} \times\{0\} \times I\right)$ gives a characteristic $G$-map for $\left.b^{n-1} \times(0,1)\right)$.

Lemma 3.2. Let $Y$ be an equivariant elementary expansion of $X$. a) Let $i: X \rightarrow Y$ be the inclusion. Then $M_{i}$ collapses equivariantly to $X$.

b) Let $r: Y \rightarrow X$ be a skeletal $G$-retraction. Then $M_{r}$ collapses equivariantly to $Y$.

Proof. a) We have $M_{i}=X \times I \cup Y \times\{1\}$ which collapses equivariantly to $X \times I$ which again collapses equivariantly to $X \times\{0\}$. 
b) By Lemma $3.1 M_{r}$ collapses equivariantly to $Y \times\{0\} \cup M_{r \mid X}=$ $Y \times\{0\} \cup X \times I$ which in turn collapses equivariantly to $Y \times\{0\}$.

Lemma 3.3. Let $f: X \rightarrow Y$ and $h: Y \rightarrow Z$ be skeletal $G$-maps. Then $M_{f} \cup_{Y} M_{h} \mathrm{~s} M_{h f}$ rel. $X \cup \dot{U} Z$.

Proof. Define $F=h p: M_{f} \rightarrow Z$, where $p: M_{f} \rightarrow Y$ is the natural projection. Then $F$ is skeletal. Consider the mapping cylinder $M_{F}$. First observe that by Lemma $1.8 M_{F}$ collapses equivariantly to $M_{F \mid X}=$ $M_{h f}$. Secondly observe that since $M_{f}$ collapses equivariantly to $Y$ (by Corollary 1.9) and since $F$ restricted to $Y$ equals $h: Y \rightarrow Z$, it follows by Lemma 3.1 that $M_{F}$ collapses equivariantly to $M_{f} \cup_{Y} M_{h}$. The claim now follows from these two facts.

By repeated use of Lemma 3.3 and the relativity principle we get.

Corollary 3.4. Let $X_{0} \stackrel{f_{1}}{\rightarrow} X_{1} \rightarrow \ldots \stackrel{f_{p}}{\rightarrow} X_{p}$ be a sequence of skeletal $G$-maps, and denote $f=f_{p} \ldots f_{1}$. Then $. I_{f} \mathrm{~s}, I_{f_{1}} \cup_{X_{1}}, I_{f_{3}} \cup \ldots \cup_{X_{p-1}} M_{f_{p}}$.

Proposition 3.5. Let $f_{0}, f_{1}: X \rightarrow Y$ be $G$-homotopic skeletal $G$-maps. Then $M_{f_{0}} \mathrm{~s} M_{f_{1}}$ rel. $X \dot{\cup} Y$. Thus, if $f_{0}$ and $f_{1}$ furthermore are $G$ homotopy equivalences, we have $s\left(M_{f_{0}}, X\right)=s\left(M_{f_{1}}, X\right) \in W h_{G}(X)$.

Proof. Let $F: X \times I \rightarrow Y$ be a $G$-homotopy from $f_{0}$ to $f_{1}$. By the equivariant skeletal approximation theorem, Corollary 1.6 in Chapter I, we can assume that $F$ is skeletal. Since $X \times I$ collapses equivariantly to $X \times\{0\}$ and to $X \times\{1\}$ it follows by Proposition 3.1 that we in particular have

$$
X \times I \cup M_{f_{0}} \mathrm{~s} I_{F} \mathrm{~s} X \times I \cup M_{f_{1}} \text { rel. } X \times I \cup Y .
$$

Let $q: X \times I \dot{\cup} Y \rightarrow X \dot{\cup} Y$ be the map defined by $q(x, t)=x$ and $q(y)=y$, and denote $\bar{M}_{F}=(X \cup \dot{\cup} Y) \cup_{q} M_{F}$. By the relativity principle, Lemma 1.6, we then have $M_{f_{0}} \mathrm{~s} \bar{M}_{F} \mathrm{~s} M_{f_{1}}$ rel. $X \dot{\cup} Y$.

Theorem 3.6. Let $f: X \rightarrow Y$ be a $G$-map. Then the following three statements are equivalent:

(a) $f$ is an equivariant simple-homotopy equiralence.

(b) There exists an equivariant skeletal approximation $\hat{f}$ to $f$ such that $M_{\hat{f}} \mathrm{~s} X$ rel. $X$.

(c) For any equivariant skeletal approximation $\tilde{f}$ to $f$ we have $M_{\tilde{f}} \mathrm{~s} X$ rel. $X$.

Proof. By Proposition 3.5 (and the fact that equivariant skeletal approximations exist) statements (b) and (c) are equivalent. We shall show that (a) and (b) are equivalent. Assume that $f: X \rightarrow Y$ is an equiv- 
ariant simple-homotopy equivalence. This means, by definition, that $f$ is $G$-homotopic to an equivariant formal deformation $k=k_{p} \ldots k_{1}: X=$ $X_{0} \rightarrow X_{1} \rightarrow \ldots \rightarrow X_{p}=Y$. Then $k$ is an equivariant skeletal approximation to $f$, and by Corollary 3.4 and repeated use of Lemma 3.2 a) and b) we have $M_{k} \mathrm{~s} X$ rel. $X$.

It remains to prove that (b) implies (a). Let $\hat{f}: X \rightarrow Y$ be an equivariant skeletal approximation to $f$ such that $M_{\hat{f}}$ s $X$ rel. $X$. We have $\hat{f}: p i: X \rightarrow M_{\hat{f}} \rightarrow Y$, where $i$ denotes the inclusion and $p$ is the natural projection. By Lemma $1.2 i$ is an equivariant simple-homotopy equivalence. By Corollary 1.9 and Lemma $1.2 p$ is an equivariant simple-homotopy equivalence. Since $f$ is $G$-homotopic to $\hat{f}=p i$ it follows that $f$ is an equivariant simple-homotopy equivalence.

Now let $f: X \rightarrow Y$ be a skeletal $G$-homotopy equivalence. We define

$$
\tau_{g}(f)=s\left(H_{f}, \mathrm{X}\right) \in W h_{G}(\mathrm{X}) .
$$

(Here the "g" in $\tau_{g}$ stands for "geometric»).) We call $\tau_{g}(f)$ for the (geometric) equivariant Whitehead torsion of $f$. If $f_{0}, f_{1}: X \rightarrow Y$ are $G$-homotopic skeletal $G$-homotopy equivalences then, by Proposition 3.5, we have $\tau_{g}\left(f_{0}\right)=\tau_{g}\left(f_{1}\right)$.

Thus we can extend the above definition to any $G$-homotopy equivalence $f: X \rightarrow Y$ by defining

$$
\tau_{g}(f)=\tau_{g}(\hat{f})
$$

where $\hat{f}$ is any equivariant skeletal approximation to $f$. We can now reformulate Theorem 3.6. in the following form.

Theorem 3.6'. A $G$-homotopy equivalence $f: X \rightarrow Y$ is an equivariant simple-homotopy equivalence if and only if $\tau_{g}(f)=0$.

In the "classical» ordinary case the question whether a homeomorphism between two $C W$ complexes is a simple-homotopy equivalence has until very recently been an open problem ever since Whitehead posed the question (see the Introduction in Whitehead [18]). An affirmative answer to this 》classical» question has now been given by Chapman [5]. He has proved the following theorem.

Theorem. (Chapman). Let $K, L$ be finite connected $C W$ complexes and let $f:|K| \rightarrow|L|$ be a map. Then $f$ is a simple-homotopy equivalence if and only if the map

$$
f \times \text { id }:|K| \times Q \rightarrow|L| \times Q
$$

is homotopic to a homeomorphism of $|K| \times Q$ onto $|L| \times Q$. 
(We have used Chapmans notation.) Here $Q$ denotes the Hilbert cube. The »only if» part is due to West [16].

In the case when $G$ is assumed to act freely we have

Theorem 3.7. Let $X$ and $Y$ be equivariant $C W$ complexes such that $G$ acts freely on $X$ and $Y$. Then any $G$-homeomorphism $f: X \rightarrow Y$ is an equivariant simple-homotopy equivalence.

Proof. Let $f: X \rightarrow Y$ be a $G$-homeomorphism. Denote the induced map on the orbit spaces by $f^{\prime}: G \backslash X \rightarrow G \backslash Y$. Then $f^{\prime}$ is a homeomorphism and hence by Chapmans result a simple-homotopy equivalence. (Observe that we have not assumed that $G \backslash X$ and $G \backslash Y$ are connected, but of course the conclusion still holds.) Thus $\tau_{g}\left(f^{\prime}\right)=0 \in W h(G \backslash X)$. By Theorem $2.7 \varphi_{*}: W h_{G}(X) \rightarrow W h(G \backslash X)$ is an isomorphism. Since $M_{f^{\prime}}=G \backslash M_{f}$ it follows that we have $\varphi_{*}\left(\tau_{g}(f)\right)=\tau_{g}\left(f^{\prime}\right)=0$. Thus $\tau_{g}(f)=0$ and hence $f$ is an equivariant simple-homotopy equivalence by Theorem $3.6^{\prime}$.

Proposition 3.8. Let $f: X \rightarrow Y$ and $h: Y \rightarrow Z$ be $G$-homotopy equivalences. Then we have

$$
\tau_{g}(h f)=\tau_{g}(f)+f_{*}^{-1} \tau_{g}(h) .
$$

Proof. By Lemma 3.3. we have $\tau_{g}(h f)=s\left(M_{h f}, X\right)=s\left(M_{f} \cup_{Y} M_{h}, X\right)$. Consider the inclusions $X \subset M_{f} \subset M_{f} \cup_{Y} M_{h}$ and let $r: M_{f} \rightarrow X$ be a $G$-retraction. By Lemma 2.2 we have

$$
\begin{aligned}
s\left(M_{f} \cup_{Y} M_{h}, X\right) & =r_{*} s\left(M_{f} \cup_{Y} M_{h}, M_{f}\right)+s\left(M_{f}, X\right) \\
& =r_{*} j_{*} s\left(M_{h}, Y\right)+s\left(M_{f}, X\right),
\end{aligned}
$$

where $j: Y \rightarrow M_{f}$ denotes the inclusion. But since $r_{j}: Y \rightarrow M_{f} \rightarrow X$ is a $G$-homotopy inverse to $f$ we have $r_{*} j_{*}=f_{*}^{-1}$. This completes the proof.

Corollary 3.9. Let $\bar{f}: Y \rightarrow X$ be a $G$-homotopy inverse to $f: X \rightarrow Y$. Then $\tau_{g}(\bar{f})=-f_{*} \tau_{g}(f)$.

Proof. We have $0=\tau_{g}(\bar{f} f)=\tau_{g}(f)+f_{*}^{-1} \tau_{g}(\bar{f})$, and hence $\tau(\bar{f})=$ $-f_{*} \tau_{g}(f)$.

Corollary 3.10. Let $f: X \rightarrow Y_{1}$ and $h: X \rightarrow Y_{2}$ be $G$-homotopy equivalences. Then $\tau_{g}(f)=\tau_{g}(h)$ if and only if there exists an equivariant simple-homotopy equivalence $\sigma: Y_{1} \rightarrow Y_{2}$ such that the diagram

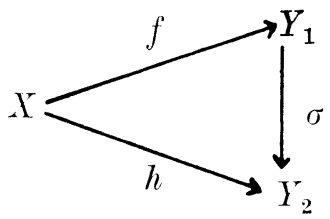

is $G$-homotopy commutative. 
Proof. Assume that such a $\sigma$ exists. Then $\tau_{g}(\sigma)=0$, and hence $\tau_{g}(h)=\tau_{g}(\sigma f)=\tau_{g}(f)+f_{*}^{-1} \tau_{g}(\sigma)=\tau_{g}(f)$.

Now assume on the other hand that $\tau_{g}(f)=\tau_{g}(h)$. Define $\sigma=$ $h \bar{f}: Y_{1} \rightarrow Y_{2}$, where $\bar{f}: Y_{1} \rightarrow X$ is a $G$-homotopy inverse to $f$. Then

$$
\tau_{g}(\sigma)=\tau_{g}(h \bar{f})=\tau_{g}(\bar{f})+(\bar{f})_{*}^{-1} \tau_{g}(h)=-f_{*} \tau_{g}(f)+f_{*} \tau_{g}(h)=0
$$

and hence $\sigma$ is an equivariant simple-homotopy equivalence.

Lemma 3.11. Let $(V, X)$ be an equivariant $C W$ pair such that the inclusion $i: X \rightarrow V$ is a $G$-homotopy equivalence. Then we have $\tau_{g}(i)=$ $s(V, X) \in W h_{G}(X)$.

Proof. By definition we have

$$
\tau_{g}(i)=s\left(M_{i}, X\right)=s(X \times I \cup V \times\{1\}, X \times\{0\}) .
$$

It follows from Corollary 1.10 that $(X \times I \cup V \times\{1\}) \mathrm{s} V \times I \mathrm{~s} V \times\{0\}$ rel. $X \times\{0\}$, and hence $s(X \times I \cup V \times\{1\}, X \times\{0\})=s(V, X)$.

It should be observed that we could as well have defined the geometric equivariant Whitehead torsion of a $G$-homotopy equivalence $f: X \rightarrow Y$ to be $\bar{\tau}_{g}(f)=f_{*} s\left(M_{f}, X\right) \in W h_{G}(Y)$. Since $f_{*}$ is an isomorphism $\bar{\tau}_{g}(f)=0$ if and only if $\tau_{g}(f)=0$. In fact we have by Corollary 3.9 that $\bar{\tau}_{g}(f)=$ - $\tau_{g}(\bar{f})$, where $\bar{f}: Y \rightarrow X$ is a $G$-homotopy inverse to $f$. Taking $\bar{\tau}_{g}(f)$ as the definition would be in complete analogy with the definition given in Cohen [6] in the standard non-equivariant case. Our choice is by Proposition 3.8 in agreement with the point of view taken in Eckmann-Maumary [7], see 2.2 in [7].

We conclude this section by observing that a restatement of the $\gg$ sum theorem», i.e. Proposition 2.4., gives us the following important result (compare again with Stöcker [15] and Cohen [6]).

Theorem 3.12. Assume that $X=X_{1} \cup X_{2}, X_{0}=X_{1} \cap X_{2}$ and $Y=$ $Y_{1} \cup Y_{2}, Y_{0}=Y_{1} \cap Y_{2}$. Let $f: X \rightarrow Y$ be a $G$-map which restricts to $G$-homotopy equivalences $f_{k}: X_{k} \rightarrow Y_{k}$ for $k=0,1,2$. Then $f$ is a $G$-homotopy equivalence and

$$
\tau_{g}(f)=\left(i_{1}\right)_{*} \tau_{g}\left(f_{1}\right)+\left(i_{2}\right)_{*} \tau_{g}\left(f_{2}\right)-\left(i_{0}\right)_{*} \tau_{g}\left(f_{0}\right),
$$

where $i_{k}: X_{k} \rightarrow X, k=0,1,2$, denote the inclusions. In particular if each $f_{k}, k=0,1,2$, is an equivariant simple-homotopy equivalence so is $f$. 


\section{§ 4. Simplified form}

We shall in this section show that every element in $W h_{G}(X)$ can be represented by a pair $(W, X)$ which is in simplified from. This means that the equivariant cells of $W-X$ are concentrated in two consecutive dimensions $n-1$ and $n$, where $n-1 \geq 2$, plus some further purely technical conditions on the attaching $G$-maps for these equivariant cells. As the case is in the ordinary non-equivariant theory the "simplified form» result is the clue to the transition from the geometric side of the theory to the algebraic side. We shall study this transition for our equivariant Whitehead theory in Chapter III in the two cases that $G$ is either a discrete abelian group or $G$ is a torus group $T^{n}, n \geq 1$. For Whiteheads original treatment of "simplified form" in the ordinary non-equivariant case see Lemmas $13-15$ in Whitehead [18]. See also (7.4) in Cohen [6].

Lemma 4.1. Let $X$ be an equirariant $C W$ complex and let

$$
f_{0}, f_{1}: G / H \times \partial I^{n} \rightarrow X
$$

be $G$-homotopic $G$-maps such that $f_{i}\left(G / H \times \partial I^{n}\right) \subset X^{n-1}, i=0,1$. Let $Y_{i}=X \cup_{f_{i}}\left(G / H \times I^{n}\right)$, the equivariant $C W$ complex obtained by adjoining $G / H \times I^{n}$ to $X$ by $f_{i}, i=0,1$. Then $Y_{0}$ s $Y_{1}$ rel. $X$.

Proof. Let $F:\left(G / H \times \partial I^{n} \times I, G / H \times \partial I^{n} \times\{0,1\}\right) \rightarrow\left(X, X^{n-1}\right)$ be a $G$-homotopy from $f_{0}$ to $f_{1}$. It follows from the equivariant skeletal approximation theorem, Corollary 1.5 in Chapter I, that we can assume that $F$ is skeletal, i.e. that $F\left(G / H \times \partial I^{n} \times I\right) \subset X^{n} \cup X^{n-1}=X^{n}$. Define $\quad Y=X \cup_{F}\left(G / H \times I^{n} \times I\right)$, the equivariant $C W$ complex obtained by adjoining $G / H \times I^{n} \times I$ to $X$ by $F: G / H \times \partial I^{n} \times I \rightarrow X^{n}$ $\rightarrow Y$. Since the obvious inclusions $i_{0}: Y_{0} \rightarrow Y$ and $i_{1} \rightarrow Y$ both are equivariant elementary expansions the lemma follows.

Let $\left(V, V_{0}\right)$ be an equivariant $C W$ pair. By $\alpha_{n}(H)\left(V-V_{0}\right)$ we denote the number of equivariant $n$-cells of type $(H)$ in $V-V_{0}$.

Lemma 4.2. Let $\left(V_{0}, X\right)$ and $\left(W_{0}, X\right)$ be equivariant $C W$ pairs and let $k: V_{0} \rightarrow W_{0}$ be an equivariant formal deformation rel. $X$. Let $V$ be an equivariant $C W$ complex containing $V_{0}$ as a subcomplex. Then there exists an equivariant $C W$ complex $W$ containing $W_{0}$ as a subcom. plex, such that $\alpha_{n}(H)\left(W-W_{0}\right)=\alpha_{n}(H)\left(V-V_{0}\right)$ for every $n$ and $(H)$, and an equivariant formal deformation $\tilde{k}: V \rightarrow W$ rel. $X$.

Proof. By induction on the number of equivariant cells in $V-V_{0}$ and on the number of equivariant elementary deformations in $k$ it follows that 
it is enough to prove the lemma in the case when $V-V_{0}$ consists of one equivariant cell and $k: V_{0} \rightarrow W_{0}$ is an equivariant elementary deformation.

If $k: V_{0} \rightarrow W_{0}$ is an equivariant elementary expansion then its natural extension $\tilde{k}: V \rightarrow W_{0} \cup_{k} V=W_{0} \cup_{V_{0}} V$ is also an equivariant elementary expansion and $W=W_{0} \cup_{V_{0}} V$ is of the required form.

Now let $k: V_{0} \rightarrow W_{0}$ be an equivariant elementary collapse. Assume that the type of the open equivariant cell $e^{p}=V-V_{0}$ is $(H)$ and let $f: G / H \times \partial I^{p} \rightarrow V_{0}^{p-1} \rightarrow V_{0}$ be an attaching $G$-map for $\bar{e}^{p}$. Thus $V=$ $V_{0} \cup_{f}\left(G / H \times I^{p}\right)$. The $G$-map $k f: G / H \times \partial I^{p} \rightarrow W_{0}^{p-1} \rightarrow V_{0}$ is $G$ homotopic to $f$ and hence by Lemma 4.1 there exists an equivariant formal deformation $h: V \rightarrow V_{0} \cup_{k f}\left(G / H \times I^{p}\right)$ rel. $V_{0}$. The natural extension $\hat{k}: V_{0} \cup_{k f}\left(G / H \times I^{p}\right) \rightarrow W_{0} \cup_{k f}\left(G / H \times I^{p}\right) \quad$ of $\quad k: V_{0} \rightarrow W_{0}$ is an equivariant elementary collapse. Thus $\tilde{k}=\hat{k} h: V \rightarrow W_{0} \cup_{k f}$ $\left(G / H \times I^{p}\right)$ is an equivariant formal deformation rel. $X$ and $W=W_{0} \cup_{k f}$ $\left(G / H \times I^{p}\right)$ is of the required form.

Recall from [10] (see Definition 1.8 in Chapter I of [10]) that we say that a $G$-pair $(Y, B)$ satisfies condition $\pi_{m}, m \geq 0$, if for any closed subgroup $H$ of $G$ every $G$-map $f:\left(G / H \times I^{m}, G / H \times \partial I^{m}\right) \rightarrow(Y, B)$ is $G$-homotopic rel. $G / H \times \partial I^{m}$ to a $G$-map from $G / H \times I^{m}$ into $B$.

Lemma 4.3. Let $(V, X)$ be an equivariant $C W$ pair which satisfies condition $\tau_{m}$, for some $m \geq 0$, and assume that

$$
V=X \cup \cup b_{i}^{m} \cup \cup b_{i}^{m+1} \cup \ldots \cup \cup b_{i}^{r} .
$$

Then there exists an equivariant $C W$ complex $W$ of the form

$$
W=X \cup \cup e_{j}^{m+1} \cup \cup e_{j}^{m+2} \cup \ldots \cup \cup e_{i}^{\max (r, m+2)}
$$

such that $V$ s $W$ rel. $X$. In fact the number of open equivariant cells $b_{i}^{s}$ and $e_{j}^{t}$ is such that

$\alpha_{k}(H)(W-X)= \begin{cases}0 & , k \leq m, \\ \alpha_{m+1}(H)(V-X) & , k=m+1, \\ \alpha_{m}(H)(V-X)+\alpha_{m+2}(H)(V-X), & k=m+2, \\ \alpha_{k}(H)(V-X) & , k \geq m+3,\end{cases}$

for every orbit type $(H)$.

Proof. Let $f_{i}:\left(G / H_{i} \times I^{m}, G / H_{i} \times \partial I^{m}\right) \rightarrow\left(V^{m}, X^{m+1}\right) \rightarrow(V, X)$ be a charachteristic $G$-map for $b_{i}^{m}$. Using the fact that $(V, X)$ satisfies condition $\pi_{m}$ and the equivariant skeletal approximation theorem, Corollary 1.5 in Chapter I, it follows that there exists a $G$-homotopy rel. $\left(G / H_{i} \times \partial I^{m}\right)$ 


$$
F_{i}^{\prime}:\left(G / H_{i} \times I^{m}, G / H_{i} \times \partial I^{m}\right) \times I \rightarrow\left(V, X^{m-1}\right)
$$

such that $\left(F_{i}^{\prime}\right)_{0}=f_{i}$ and $\left(F_{i}^{\prime}\right)_{1}\left(G / H_{i} \times I^{m} \times\{1\}\right) \subset X^{m}$. It now follows by applying the equivariant skeletal approximation theorem once more, this time Corollary 1.6 in Chapter $I$, that we can assume that $F_{i}^{\prime}$ is skeletal, i.e. that $F_{i}^{\prime}\left(G / H_{i} \times I^{m} \times I\right) \subset V^{m+1}$. Observe that

$$
F_{i}\left(G / H_{i} \times \partial I^{m+1}\right) \subset V^{m}
$$

and $F_{i}^{\prime}\left(G / H_{i} \times J^{m}\right) \subset X^{m}$. Now define

$$
F_{i}: G / H_{i} \times J^{m+1} \rightarrow V^{m+1} \rightarrow V
$$

by $F_{i}(a, 1)=F_{i}^{\prime}(a)$, for $(a, 1) \in G / H_{i} \times I^{m+1} \times\{1\}, \quad$ and $\quad F_{i}(b, t)=$ $F_{i}^{\prime}(b), \quad$ for $(b, t) \in G / H_{i} \times \partial I^{m+1} \times I, t \in I$. For each $F_{i}$ adjoin $G / H_{i} \times I^{m+2}$ to $V$ by $F_{i}$, thus forming

$$
\tilde{V}=V \cup_{F_{i}}\left(\dot{U} G / H_{i} \times I^{m+2}\right) .
$$

Since $F_{i}$ also satisfies $F_{i}\left(G / H_{i} \times \partial I^{m+1}\right) \subset V^{m}$ it follows that $\tilde{V}$ is an equivariant expansion of $V$. Let

$$
h_{i}: G / H_{i} \times I^{m+2} \rightarrow \tilde{V}
$$

be the restriction of the natural projection. Denoting $h_{i}\left(G / H_{i} \times \stackrel{i}{I}^{m+1}\right)=$ $B_{i}^{m+1}$ and $h_{i}\left(G / H_{i} \times \stackrel{i}{I}^{m+2}\right)=B_{i}^{m+2}$ we can write

$$
\tilde{V}=V \cup \cup B_{i}^{m+1} \cup \cup B_{i}^{m+2} .
$$

Since $\dot{B}_{i}^{m+1} \subset V^{m} \subset X \cup \cup b_{i}^{m}$ it follows that

$$
\tilde{V}_{0}=X \cup \cup b_{i}^{m} \cup \cup B_{i}^{m+1}
$$

is a subcomplex of $\tilde{V}$. Let $\bar{h}_{i}: G / H_{i} \times I^{m+1} \rightarrow \tilde{V}_{0}$ denote the $G$-map obtained by restricting $h_{i}$ to $G / H_{i} \times I^{m-1} \times\{0\}$. Then $\bar{h}_{i}$ is a characteristic $G$-map for $\bar{B}_{i}^{m+1}$ and its restriction $\bar{h}_{i} \mid: G / H_{i} \times I^{m} \rightarrow \tilde{V}_{0}$ is a characteristic $G$-map for $\bar{b}_{i}^{m}$, and moreover $\bar{h}_{i}\left(G / H_{i} \times J^{m}\right)=$ $F_{i}\left(G / H_{i} \times J^{m} \times\{0\}\right)=F_{i}^{\prime}\left(G / H_{i} \times J^{m}\right) \subset X^{m}$. Thus it follows directly from the definitions that $\tilde{V}_{0}$ collapses equivariantly to $X$. By Lemma 4.2 there exists an equivariant $C W$ complex $W$ containing $X$ as a subcomplex, with $\alpha_{n}(H)(W-X)=\alpha_{n}(H)\left(\tilde{V}-\tilde{V}_{0}\right)$ for every $n$ and $(H)$, such that $\tilde{V}$ s $W$ rel. X. Since, as we already noted, $\tilde{V}$ is an equivariant expansion of $V$ it follows that $V$ s $W$ rel. $X$. Clearly the number of equivariant cells in $W-X$ with a specific dimension and type is as required. 
Corollary 4.4. Let $(V, X)$ be an equivariant $C W$ pair such that $X$ is a strong $G$-deformation retract of $V$. Then there exists an equivariant $C W$ complex $W$ of the form

$$
W=X \cup \cup b_{i}^{n-1} \cup \cup b_{i}^{n}, \text { where } n-1 \geq 2,
$$

such that $V$ s $W$ rel. $X$ and such that there are characteristic $G$-maps $y_{i}: G / H_{i} \times I^{n-1} \rightarrow \bar{b}_{i}^{n-1}$ satisfying $h_{i}\left(\left\{e H_{i}\right\} \times \partial I^{n-1}\right)=\left\{y_{i}\right\}, y_{i} \in X$, and $f_{i}: G / H_{i} \times I^{n} \rightarrow \bar{b}_{i}^{n}$ satisfying $f_{i}\left(\left\{e H_{i}\right\} \times J^{n-1}\right)=\left\{x_{i}\right\}, x_{i} \in X$.

Moreover, for any closed subgroup $H$ of $G$ and any $G$-component $G W_{1}^{H}$ of $G W^{H}$, we have $\alpha_{n-1}(H)\left(G W_{1}^{H}-G X_{1}^{H}\right)=\alpha_{n}(H)\left(G W_{1}^{H}-G X_{1}^{H}\right)$ where $G X_{1}^{H}$ denotes $G$-component corresponding to $G W_{1}^{H}$.

Proof. Since $X$ is a strong $G$-deformation retract of $V$ it follows that $(V, X)$ satisfies condition $\pi_{m}$ for all $m \geq 0$. Thus, by repeated use of Lemma 4.3, there exists $W^{\prime}$ such that $W^{\prime}=X \cup \cup e_{i}^{n-1} \cup \cup e_{i}^{n}$, with $n-1 \geq 2$, and $V \mathrm{~s} W^{\prime}$ rel. $X$. Let $h_{i}^{\prime}: G / H_{i} \times I^{n-1} \rightarrow \bar{e}_{i}^{n-1}$ be characteristic $G$-maps. Since $r h_{i}^{\prime}: G / H_{i} \times I^{n-1} \rightarrow X$, where $r: W^{\prime} \rightarrow X$ is a $G$-retraction, is an extension of $h_{i}^{\prime}: G / H_{i} \times \partial I^{n-1} \rightarrow X$ it follows that $h_{i}^{\prime} \mid$ is $G$-homotopic the $G$-map which maps $\left\{e H_{i}\right\} \times \partial I^{n-1}$ to some point $y_{i} \in X$. Adjoining $G / H_{i} \times I^{n-1}$ to $X$ by these "equivariantly constant» maps we obtain an equivariant $C W$ complex $X \cup \cup b_{i}^{n-1}$, and it follows from Lemma 4.1 that $\left(X \cup \cup e_{i}^{n-1}\right) \mathrm{s}\left(X \cup \cup b_{i}^{n-1}\right)$ rel. $X$. By Lemma 4.2 there exists $\widetilde{W}=X \cup \cup b_{i}^{n-1} \cup \cup d_{i}^{n}$ such that $W^{\prime} \mathrm{s} \widetilde{W}$ rel. $X$. Let $\tilde{f}_{i}$ : $G / H_{i} \times \partial I^{n} \rightarrow \dot{d}_{i}^{n} \rightarrow X \cup \cup b_{i}^{n-1}$ be attaching $G$-maps. Since $J^{n-1}$ is contractible it follows that $\tilde{f}_{i}$ is $G$-homotopic to some $f_{i}: G / H_{i}$ 冫 $\partial I^{n}$ $\rightarrow X \cup \cup b_{i}^{n-1}$ satisfying $f_{i}\left(\left\{e H_{i}\right\} \times J^{n-1}\right)=\left\{x_{i}\right\} \in X$. Adjoining $G / H_{i} \times$ $I^{n}$ to $X \cup \cup b_{i}^{n-1}$ by the $f_{i}: \mathrm{s}$ we obtain $W=X \cup \cup b_{i}^{n-1} \cup \cup b_{i}^{n}$, and by Lemma 4.1 it follows that $\widetilde{W} \mathrm{~s} W$ rel. $X$. (Now denote the corresponding characteristic $G$-maps for $\bar{b}_{i}^{n}$ also by $f_{i}$ ).

To prove the claim about the number of the equivariant cells $b_{i}^{n-1}$ and $b_{i}^{n}$ observe that the $G$-homotopy equivalence $i: X \rightarrow W$ induces a oneto-one correspondence between the $G$-components of $G X^{H}$ and $G W^{H}$, and the restriction $i \mid: G X_{1}^{H} \rightarrow G W_{1}^{H}$ is again a $G$-homotopy equivalence. Let, for convenience, $\hat{i}: A \rightarrow B$ denote the inclusion induced by $i$ on the orbit spaces. Since $B-A$ consists of $(n-1)$-cells and $n$-cells and since $\hat{i}: A \rightarrow B$ is a homotopy equivalence it follows that the number of $(n-1)$-cells and $n$-cells in $B-A$ are the same. This being true for any $G$-component $G W_{1}^{H}$, the claim follows by induction starting from $H=G$, i.e. from the components of the fixed point set. 


\section{Chapter III. Actions of abelian groups}

\section{$\S 1$. Actions of discrete abelian groups}

In this section the transformation group $G$ is assumed to be a discrete abelian group. First we recall the definition of the Whitehead group $W h(\pi)$ of a group $\pi$ and some facts about torsion of acyclic chain complexes of based modules, see Whitehead [18], Milnor [14], Maumary [13] and Cohen [6].

Let $\pi$ be a group and $R=Z[\pi]$ the group ring of $\pi$ over the integers. Denote the group of all non-singular $n \times n$ matrices over $R$ by $G L(n, R)$. We have the natural inclusion of $G L(n+1, R)$ into $G L(n, R)$ given by

$$
\mathrm{A} \rightarrow\left(\begin{array}{ll}
\mathrm{A} & 0 \\
0 & 1
\end{array}\right)
$$

The direct limit $G L(R)=\lim _{\rightarrow} G L(n, R)$ is called the infinite general linear group of $R$. A matrix is called elementary if it agrees with the identity matrix except for one off-diagonal entry. Let $E(R)$ denote the subgroup of $G L(R)$ generated by all elementary matrices. Whitehead proved that $E(R)$ is the commutator subgroup of $G L(R)$. Let $F(R)$ be the subgroup of $G L(R)$ generated by $E(R)$ and all matrices obtained by replacing one diagonal entry in an identity matrix by $\pm \alpha$, where $\alpha \in \pi$. Define

$$
W h(\pi)=G L(R) / F(R) .
$$

This is the Whitehead group of $\pi$. Since $F(R)$ contains the commutator subgroup of $G L(R)$ it follows that $W h(\tau)$ is in fact a group and moreover that it is abelian. We write $W h(\tau)$ additively. Denote the natural projection by $\tau: G L(R) \rightarrow W h(\tau)$, and identifying a non-singular $n \times n$ matrix $A$ with its image in $G L(R)$ we write $\tau(A) \in W h(\tau)$ and call $\tau(A)$ for the torsion of the matrix $A$. Thus we have $\tau(A B)=\tau(A)+\tau(B)$.

Let $A$ be an $n \times n$ matrix over $R=Z[\tau]$. Then $\tau(A)=0 \in W h(\tau)$ if and only if $A$ can be transformed into an identity matrix $I^{n+p}$ by a finite sequence of operations of the following type.

(1) Multiply a row by -1 .

(2) Multiply a row on the left by an element of $\pi$.

(3) Change a row by adding to it some other row.

(4) Expand to $\left(\begin{array}{ll}\mathrm{A} & 0 \\ 0 & 1\end{array}\right) \in G L(n+1, R)$.

Observe that a permutation of the rows can be performed by using operations of type (1) and (3). Also observe that the operation of changing 
a row by adding to it a left group ring multiple of some other row is the composite of operations of type (1), (2) and (3). In fact this operation is the result of multiplying the matrix $A$ on the left by an elementary matrix, but it is convenient to have the above four operations as the basic ones.

Let $M$ be a free $Z[\pi]$-module. (Here and in the following we always mean by "free module» a »finite dimensional free module» if not otherwise is explicitly stated.) Let $\left\{e_{\mathbf{1}}, \ldots, e_{m}\right\}$ be a basis for $M$. The family of preferred bases generated by $\left\{e_{1}, \ldots, e_{m}\right\}$ is the family of all bases $\left\{e_{1}^{\prime}, \ldots, e_{m}^{\prime}\right\}$ such that the change of bases matrix $A=\left(a_{i j}\right)$, where $e_{i}^{\prime}=\sum_{j} a_{i j} e_{j}, a_{i j} \in Z[\pi]$, satisfies $\tau(A)=0 \in W h(\pi)$. (Any group ring $Z[\pi]$ has the property that any two bases of a free $Z[\pi]$-module contain the same number of elements, i.e. the dimension is well-defined.) A free $Z[\pi]$-module together with a family of preferred bases is called a based $Z[\pi]$-module.

Now let $M$ and $N$ be based $Z[\pi]$-modules and

$$
f: M \rightarrow N
$$

an isomorphism of $Z[\tau]$-modules. Let $B$ denote the matrix of $f$ with respect to some bases for $M$ and $N$ from the respective families of preferred bases. The element $\tau(B) \in W h(\tau)$ is independent of which bases from the families of preferred bases one chooses and is called the torsion of the isomorphism $f: M \rightarrow N$ and denoted by

$$
\tau(f) \in W h(\pi)
$$

Finally let us recall the definition of the torsion of an acyclic chain complex of based $Z[\pi]$-modules. Let

$$
C: 0 \rightarrow C_{n} \stackrel{\partial}{\rightarrow} C_{n-1} \stackrel{\partial}{\rightarrow} \cdots \stackrel{\partial}{\rightarrow} C_{0} \rightarrow 0
$$

be an acyclic chain complex over $Z[\tau]$ where each $C_{i}$ is a based $Z[\tau]$ module. Since $C$ is acyclic and each $C_{i}$ is a free module there exists a chain homotopy $\delta: C \rightarrow C$ from the identity homomorphism to the zero homomorphism (such a $\delta$ is also called a chain contraction), that is, homomorphisms $\delta: C_{i} \rightarrow C_{i+1}$ such that $\delta \partial+\partial \delta=\mathrm{id}$. (The homomorphisms $\delta$ can be chosen such that $\delta^{2}=0$, which is a convenient but not necessary choice.) Denote

$$
\begin{aligned}
& C_{\text {odd }}=C_{1} \oplus C_{3} \oplus \ldots \\
& C_{\text {even }}=C_{0} \oplus C_{2} \oplus \ldots
\end{aligned}
$$

and consider $C_{\text {odd }}$ and $C_{\text {even }}$ as based $Z[\pi]$-modules in the obvious way. Now the homomorphisms $\partial$ and $\delta$ define an isomorphism 


$$
(\partial+\delta): C_{\text {odd }} \rightarrow C_{\text {even }}
$$

and $\tau(\partial+\delta) \in W h(\pi)$ depends only on $C$ and not on the different choices made. The torsion of $C$ is now defined to be

$$
\tau(C)=\tau(\partial+\delta) \in W h(\pi) .
$$

Observe that if $C$ is of the form

$$
C: 0 \rightarrow C_{n} \stackrel{\partial}{\stackrel{\partial}{\Rightarrow}} C_{n-1} \rightarrow 0
$$

then we have

$$
\tau(C)=(-1)^{n-1} \tau(\partial) .
$$

This fact is of great importance for us as well as the following result. If

$$
0 \rightarrow C^{\prime} \stackrel{i}{\rightarrow} C \stackrel{j}{\rightarrow} C^{\prime \prime} \rightarrow 0
$$

is a short exact sequence of acyclic chain complexes of based $Z[\pi]$-modules such that in each degree $m$ the short exact sequence

$$
0 \rightarrow C_{m}^{\prime} \stackrel{i}{\rightarrow} C_{m} \rightarrow C_{m}^{\prime \prime} \rightarrow 0
$$

splits as a sequence of based $Z[\pi]$-modules (i.e. the union of a preferred basis for $C_{m}^{\prime}$ and one for $C_{m}^{\prime \prime}$ is a preferred basis for $C_{m}$ ) then

$$
\tau(C)=\tau\left(C^{\prime}\right)+\tau\left(C^{\prime \prime}\right) .
$$

With this much about torsion of acyclic chain of based modules in mind we are now ready to proceed.

Let $Y$ be an equivariant $C W$ complex and $H$ a subgroup of $G$. Consider $Y^{H}$, i.e. the set of points fixed under $H$. Since $G$ is assumed to be abelian we have $G Y^{H}=Y^{H}$. Let $Y_{1}^{H}$ be a component of $Y^{H}$. Denote $\alpha=I_{1}^{H}$ and define

$$
G_{\alpha}=\left\{g \in G \mid g Y_{1}^{H}=Y_{1}^{H}\right\} .
$$

We call $G_{\alpha}$ for the group of $Y_{1}^{H}$. Observe that $G_{\alpha}$ is a proper subgroup of $G$ if and only if the $G$-component $G Y_{1}^{H}$ contains more than one connected component. Since every point in $Y_{1}^{H}$ is fixed under $H$ it follows that $Y_{1}^{H}$ is a $\left(G_{\alpha} / H\right)$-equivariant $C W$ complex. Also observe that if $Y_{2}^{H}$ is a component of $Y^{H}$ belonging to the same $G$-component as $Y_{1}^{H}$, i.e. $G Y_{2}^{H}=G Y_{1}^{H}$, then the group of $Y_{2}^{H}$ equals $G_{\alpha}$ and $Y_{2}^{H}$ and $Y_{1}^{H}$ are isomorphic as $\left(G_{\alpha} / H\right)$-equivariant $C W$ complexes. Now define

$$
Y_{1}^{>H}=\bigcup Y_{i}^{K}
$$

where the union is over all subgroups $K$ such that $H \subset K$ and $H \neq K$, and for fixed $K$ the union is over all components $Y_{i}^{K}$ of $Y^{K}$ which are 
subsets of $Y_{1}^{H}$. We have $G_{\alpha} Y_{1}^{>H}=Y_{1}^{>H}$, (the groups of all the components $Y_{i}^{K}$ may well be proper subgroups of $\left.G_{\alpha}\right)$. Thus $Y_{1}^{>H}$ is a $\left(G_{\alpha} / H\right)$ equivariant subcomplex of $Y_{1}^{H}$ and $G_{\alpha} / H$ acts freely on $Y_{1}^{H}-Y_{1}^{>H}$. (Of course $Y_{1}^{H}-Y_{1}^{>H}$ is empty for all but a finite number of subgroups $H$ but an action on the empty set is free.)

Now let $(V, X)$ be an equivariant $C W$ pair such that $i: X \rightarrow V$ is a $G$-homotopy equivalence. Then $i$ induces an one-to-one correspondence between the components of $X^{H}$ and $V^{H}$ for every subgroup $H$. Let $X_{1}^{H}$ be a component of $X^{H}$ and let $V_{1}^{H}$ denote the corresponding component $V^{H}$. Denote the group of $X_{1}^{H}$ by $G_{\alpha}$ and observe that $G_{\alpha}$ is also the group of $V_{1}^{H}$. Thus we have the $\left(G_{\alpha} / H\right)$-equivariant $C W$ pair $\left(V_{1}^{H}, X_{1}^{H} \cup V_{1}^{>H}\right)$. If $X_{2}^{H}$ is a component of $X^{H}$ belonging to the same $G$-component as $X_{1}^{H}$ then the $\left(G_{\alpha} / H\right)$-equivariant $C W$ pairs $\left(V_{2}^{H}, X_{2}^{H} \cup V_{2}^{>H}\right)$ and $\left(V_{1}^{H}, X_{1}^{H} \cup V_{1}^{>H}\right)$ are isomorphic. Let

$$
\cdots \rightarrow C_{n}\left(V_{1}^{H}, X_{1}^{H} \cup V_{1}^{>H}\right) \stackrel{\partial}{\rightarrow} C_{n-1}\left(V_{1}^{H}, X_{1}^{H} \cup V_{1}^{>H}\right) \rightarrow \cdots
$$

be the cellular chain complex of $\left(V_{1}^{H}, X_{1}^{H} \cup V_{1}^{>H}\right)$. That is, $C_{n}\left(V_{1}^{H}\right.$, $\left.X_{1}^{H} \cup V_{1}^{>H}\right)=H_{n}\left(\left(V_{1}^{H}\right)^{n} \cup X_{1}^{H} \cup V_{1}^{>H},\left(V_{1}^{H}\right)^{n-1} \cup X_{1}^{H} \cup V_{1}^{>H}\right)$, where $H_{n}($,$) denotes singular homology with integer coefficients, and \partial$ is the boundary homomorphism in the exact homology sequence of the corresponding triple. Since $G_{\alpha} / H$ acts freely on $V_{1}^{H}-\left(X_{1}^{H} \cup V_{1}^{>H}\right)$ it follows that each $C_{n}\left(V_{1}^{H}, X_{1}^{H} \cup V_{1}^{>H}\right)$ is a free $Z\left[G_{\alpha} / H\right]$-module and a basis is obtained by choosing one ordinary $n$-cell from each $\left(G_{\alpha} / H\right)$ equivariant $n$-cell of $V_{1}^{H}-\left(X_{1}^{H} \cup V_{1}^{>H}\right)$. Since any two bases obtained in this way differ from each other only in the order of the bases elements and by multiplication of the bases elements by \pm elements from the group $G_{\alpha} / H$, it follows that they both generate the same family of preferred bases. Thus each $C_{n}\left(V_{1}^{H}, X_{1}^{H} \cup V_{1}^{>H}\right)$ becomes a based $Z\left[G_{\alpha} H\right]$-module in this way. Since the homology of the chain complex $C\left(\Gamma_{1}^{H}, X_{1}^{H} \cup V_{1}^{>H}\right)$ is isomorphic to $H_{*}\left(V_{1}^{H}, X_{1}^{H} \cup V_{1}^{>H}\right)$ it follows from the lemma below that $C\left(V_{1}^{H}, X_{1}^{H} \cup V_{1}^{>H}\right)$ is an acyclic chain complex.

Lemma 1.1. Let $(Y, B)$ be an equivariant $C H$ pair and let $\left\{Y_{1}, \ldots, Y_{m}\right\}$ be a finite collection of equivariant subcomplexes of $Y$ which is closed under intersection. If the inclusions $i: B \rightarrow Y$ and $i_{k}: B \cap Y_{k} \rightarrow Y_{k}, k=1, \ldots, m$, are $G$-homotopy equivalences then so is the inclusion $j: B \cup\left(\bigcup_{k=1}^{m} Y_{k}\right) \rightarrow Y$.

Proof. We shall prove by induction on $m$ that $\hat{i}: B \rightarrow B \cup\left(\bigcup_{k=1}^{m} Y_{k}\right)$ is a $G$-homotopy equivalence, and since $i: B \rightarrow Y$ is a $G$-homotopy equivalence the claim follows from this. Let $m=1$. Since $i_{1}: B \cap Y_{1} \rightarrow Y_{1}$ 
is a $G$-homotopy equivalence $B \cap Y_{1}$ is a strong $G$-deformation retract of $Y_{1}$ and hence $B$ is a strong $G$-deformation retract of $B \cup Y_{1}$ which shows that $\hat{i}: B \rightarrow B \cup Y_{1}$ is a $G$-homotopy equivalence.

Now assume that our claim is true for the value $m-1$ and denote $\tilde{Y}_{m-1}=\bigcup_{k=1}^{m} Y_{k}$. Since the family of the $Y_{k}: \mathrm{s}$ is closed under intersection it follows that $\tilde{Y}_{m-1} \cap Y_{m}$ is the union of $m-1$ sets $Y_{k}$. Thus by the induction hypothesis the inclusions $\hat{i}: B \rightarrow B \cup\left(\tilde{Y}_{m-1} \cap Y_{m}\right)$ and $\hat{i}: B \rightarrow B \cup \widetilde{Y}_{m-1}$ are $G$-homotopy equivalences and so is also $\hat{i}$ : $B \rightarrow B \cup Y_{m}$. Thus it follows that the inclusions of $B \cup\left(\tilde{Y}_{m-1} \cap Y_{m}\right)$ into both $B \cup \tilde{Y}_{m-1}$ and $B \cup Y_{m}$ are $G$-homotopy equivalences. It now follows that $B \cup\left(\tilde{Y}_{m-1} \cap Y_{m}\right)$ is a strong $G$-deformation retract of $B \cup\left(\tilde{Y}_{m-1} \cup Y_{m}\right)$, and therefore the composite $\hat{i}: B \rightarrow B \cup$ $\left(\tilde{Y}_{m-1} \cap Y_{m}\right) \rightarrow B \cup\left(\tilde{Y}_{m-1} \cup Y_{m}\right)$ is $G$-homotopy equivalence.

We now apply Lemma 1.1 as follows. Since $V^{H}$ is a finite equivariant $C W$ complex and $G$ is abelian there exists a finite number of subgroups $K_{1}, \ldots, K_{m}$ such that if $K$ is any subgroup of $G$ such that $H \subset K$ and $H \neq K$ then $Y^{K}=Y^{K_{i}}$ for some $i=1, \ldots, m$. In other words let $K_{1}, \ldots, K_{m}$ be the family of all isotropy groups for points in $V^{H}$ except the group $H$, which of course may or may not occur as the isotropy group of a point in $V^{H}$. Since $V^{K_{i}} \cap V^{K_{j}}=V^{\left(K_{i}+K_{j}\right)}$ it follows that the family $\left\{V^{K_{1}}, \ldots, V^{K_{m}}\right\}$ is closed under intersection. Since $i: X \rightarrow V$ is a $G$-homotopy equivalence it follows that also $i \mid: X^{H} \rightarrow V^{H}$ and $i \mid: X^{H} \cap V^{K_{i}}=X^{K_{i}} \rightarrow V^{K_{i}}$ are $G$-homotopy equivalences. Thus by Lemma 1.1. the inclusion $j: X^{H} \cup\left(\bigcup_{i=1}^{m} V^{K_{i}}\right) \rightarrow Y^{H}$ is a $G$-homotopy equivalence, and hence in particular a homotopy equivalence. Since the component of $X^{H} \cup\left(\bigcup_{i=1}^{m} V^{K_{i}}\right)$ which contains $X_{1}^{H}$ equals $X_{1}^{H} \cup V_{1}^{>H}$ the restriction of $j$ gives a homotopy equivalence $j \mid: X_{1}^{H} \cup V_{1}^{>H} \rightarrow V_{1}^{H}$. Thus $H_{*}\left(V_{1}^{H}, X_{1}^{H} \cup V_{1}^{>H}\right)=0$ and the chain complex $C\left(V_{1}^{H}, X_{1}^{H} \cup V_{1}^{>H}\right)$ is acyclic. We have proved.

Proposition 1.2. Let $(V, X)$ be an equirariant $C W$ pair such that $i: X \rightarrow V$ is a $G$-homotopy equivalence and let $H$ be any subgroup of $G$ and $X_{1}^{H}$ a component of $X^{H}$. Denoting the group of $X_{1}^{H}$ by $G_{\alpha}$ we have that $C\left(V_{1}^{H}, X_{1}^{H} \cup V_{1}^{>H}\right)$ is an acyclic chain complex of based $Z\left[G_{\alpha} / H\right]$-modules.

We denote the torsion of the chain complex $C\left(V_{1}^{H}, X_{1}^{H} \cup V_{1}^{>H}\right)$ by

$$
\tau\left(C(V, X)_{1}^{H}\right) \in W h\left(G_{\alpha} / H\right) .
$$


Next we show that this torsion is an invariant of equivariant simplehomotopy type.

Proposition 1.3. Assume that $V$ s $W$ rel. $X$. Then we have

$$
\tau\left(C(V, X)_{1}^{H}\right)=\tau\left(C(W, X)_{1}^{H}\right)
$$

for every subgroup $H$ of $G$ and any component $X_{1}^{H}$ of $X^{H}$.

Proof. It is enough to prove this for the case that $W$ is equivariant elementary expansion of $V$. Thus assume that this is the case and denote

$$
W=V \cup b^{n-1} \cup b^{n} .
$$

Assume that the type of this equivariant elementary expansion is $K$ and let

$$
\sigma: G / K \times I^{n} \rightarrow W
$$

be a characteristic simple $G$-map for $\left(b^{n}, b^{n-1}\right)$. Since the set $\sigma\left(\{e K\} \times J^{n-1}\right)$ is connected it lies in one component, say $X_{0}^{K}$, of $X^{K}$.

If $K \subset H$ then $W^{\boldsymbol{H}}=V^{\boldsymbol{H}}$ and the claim is obvious. If $H \subset K$, where $H \neq K$, and $G X_{0}^{K} \subset G X_{1}^{H}$, then by excision we have an isomorphism of chain complexes $i: C\left(V_{1}^{H}, X_{1}^{H} \cup V_{1}^{>H}\right) \cong C\left(W_{1}^{H}, X_{1}^{H} \cup W_{1}^{>H}\right)$ which in each degree is an isomorphism of based modules and hence the wanted conclusion follows. In case $G X_{0}^{K} \nsubseteq G X_{1}^{H}$ we have again the situation that $G W_{1}^{H}=G V_{1}^{H}$ and this is also true for $K=H$.

Thus it only remains to prove our claim in the case $H=K$ and for the component $X_{0}^{K}$ (or any other component of the $G$-component $G X_{0}^{K}$ ). Denote $C^{\prime}=C\left(V_{0}^{K}, X_{0}^{K} \cup V_{0}^{>K}\right) \quad$ and $\quad C=C\left(W_{0}^{K}, X_{0}^{K} \cup W_{0}^{>K}\right)$, and let $G_{\beta}$ be the group of $X_{0}^{K}$. Then we have a short exact sequence of chain complexes

$$
0 \rightarrow C^{\prime} \rightarrow C \rightarrow C^{\prime \prime} \rightarrow 0,
$$

where $C^{\prime \prime}$ is of the form

$$
0 \rightarrow C_{n}^{\prime \prime} \stackrel{\frac{\partial}{\rightrightarrows}}{\rightrightarrows} C_{n-1}^{\prime \prime} \rightarrow 0
$$

and $C_{n}^{\prime \prime}$ and $C_{n-1}^{\prime \prime}$ are based $Z\left[G_{\beta} / K\right]$-modules of rank 1 and $\tau(\partial)=0$. Since moreover the above short exact sequence of chain complexes in each dimension splits as a sequence of based $Z\left[G_{\beta} / K\right]$-modules we have

$$
\tau(C)=\tau\left(C^{\prime}\right)+\tau\left(C^{\prime \prime}\right)=\tau\left(C^{\prime}\right)+(-1)^{n-1} \tau(\partial)=\tau\left(C^{\prime}\right),
$$

which is what we wanted to prove.

Thus we have a well-defined map

$$
\Phi: W h_{G}(X) \rightarrow \sum_{H} \sum_{i=1}^{m(H)} \oplus W h\left(G_{\alpha(i, H)} / H\right)
$$


by defining

$$
\Phi(s(V, X))=\left\{\tau\left(C(V, X)_{i}^{H}\right\}_{1 \leq i \leq m(H)}, \text { all } H \subset G .\right.
$$

Here $m(H)$ denotes the number of $G$-components of $X^{H}$ and $G_{\alpha(i, H)}$ is the group of a component $X_{i}^{H}$ representing the $G$-component $G X_{i}^{H}$, $1 \leq i \leq m(H)$. The direct sum is over all subgroups $H$ of $G$ and for each fixed $H$ over a set of one representing component from each $G$ component of $X^{H}$. This definition is independent on which component of a $G$-component we choose to represent the $G$-component. Moreover for any $(V, X)$ we have that $\tau\left(C(V, X)_{i}^{H}\right) \neq 0$ for only a finite number of subgroups $H$.

It follows from the appropriate short exact sequence of chain complexes that $\tau\left(C\left(V \cup_{X} W, X\right)_{i}^{H}\right)=\tau\left(C(V, X)_{i}^{H}\right)+\tau\left(C(W, X)_{i}^{H}\right)$, for any subgroup $H$ and any component $X_{i}^{H}$ of $X^{H}$. Thus

$$
\Phi\left(s\left(V \cup_{X} W, X\right)\right)=\Phi(s(V, X))+\Phi(s(W, X)),
$$

that is, $\Phi$ is a homomorphism. We are now ready for

Theorem 1.4. Assume that $X$ is an equivariant $C W$ complex such that for any subgroup $H$ of $G$ each component $X_{i}^{H}$ of $X^{H}$ is simply connected. Then $\Phi$ is an isomorphism.

Proof. Let us first make a general remark. Let $(X, A)$ be a pair with $X$ and $A$ connected and assume that $A$ is simply connected. Then any map $f:\left(I^{n}, \partial I^{n}\right) \rightarrow(X, A)$ with $f\left(J^{n-1}\right)=\{a\}$, some a $\in A$, determines a unique element $[f] \in \tau_{n}(X, A)$, in other words we need not consider any base point in $A$. It follows that if $(X, A)$ is a $\pi$-pair where $\pi$ is a discrete group then $\pi_{n}(X, A)$ is a left $Z[\pi]$-module, and moreover the Hurewicz homomorphism $\phi: \pi_{n}(X, A) \rightarrow H_{n}(X, A)$ is a homomorphism of $Z[\pi]$-modules.

Let $s(W, X) \in W h_{G}(X)$ be such that $\Phi(s(W, X))=0$. By Corollary 4.4 in Chapter II (and the fact that $\Phi$ is well-defined, i.e. Proposition 1.3.) we can assume that $(H, X)$ is in simplified form. Thus we have

$$
W=X \cup \cup b_{i}^{n-1} \cup \cup b_{i}^{n}, \text { where } n-1 \geq 2 .
$$

We denote

$$
Y=X \cup \cup b_{i}^{n-1} .
$$

Now let $H$ denote a subgroup of $G$ which occurs as the type of some of the equivariant cells $b_{i}^{n}$. Let $X_{1}^{H}$ be a component of $X^{H}$ and let $Y_{1}^{H}$ and $W_{1}^{H}$ be the corresponding components of $Y^{H}$ and $W^{H}$, respectively. By the second part of Corollary 4.4. in Chapter II the number of equivariant $n$-cells $b_{i}^{n}$ in $G W_{1}^{H}$ which have type $H$ equals the number of equivariant 
$(n-1)$-cells $b_{i}^{n-1}$ in $G W_{1}^{H}$ (and hence in $G Y_{1}^{H}$ ) which have type $H$. Let us denote these by $b_{s}^{n}$ and $b_{s}^{n-1}, s=1, \ldots, m$. Thus we have

$$
\begin{aligned}
& G W_{1}^{H}=G\left(Y_{1}^{H} \cup W_{\mathrm{I}}^{>H}\right) \cup \bigcup_{s=1}^{m} b_{s}^{n}, \\
& G Y_{1}^{H}=G\left(X_{1}^{H} \cup Y_{1}^{>H}\right) \cup \bigcup_{s=1}^{m} b_{s}^{n-1} .
\end{aligned}
$$

Now let $G_{\alpha}$ be the group of $X_{1}^{H}$, and hence also the group of $Y_{1}^{H}$ and $W_{1}^{H}$, and consider the $\left(G_{\alpha} / H\right)$-equivariant $C W$ pair $\left(W_{1}^{H}, X_{1}^{H} \cup W_{1}^{>H}\right)$. We have the commutative diagram

$$
\begin{aligned}
& 0 \rightarrow H_{n}\left(W_{1}^{H}, Y_{1}^{H} \cup W_{1}^{>H}\right) \stackrel{0}{\stackrel{亏}{\rightrightarrows}} H_{n-1}\left(Y_{1}^{H} \cup{W_{1}^{>H}}_{1}, X_{1}^{H} \cup W_{1}^{>H}\right) \rightarrow 0 \\
& \begin{array}{cc}
\uparrow & \phi \\
\pi_{n}\left(W_{1}^{H}, Y_{1}^{H} \cup W_{1}^{>H}\right) \rightarrow \pi_{n-1}\left(Y_{1}^{H} \cup W_{1}^{>H}, X_{1}^{H} \cup W_{1}^{>H}\right)
\end{array}
\end{aligned}
$$

where $\phi$ denotes the Hurewicz homomorphism. First observe that the upper row equals the cellular chain complex of $\left(W_{1}^{H}, X_{1}^{H} \cup W_{1}^{>H}\right)$. Thus we have

$$
(-1)^{n-1} \tau(\partial)=\tau\left(C(W, X)_{1}^{H}\right)=0 \in W h\left(G_{\alpha} / H\right) .
$$

Secondly observe that since $X_{1}^{H}$ is simply connected by assumption and $X_{1}^{H} \cup W_{1}^{>H}$ is obtained from $X_{1}^{H}$ by adjoining (ordinary) $(n-1)$-cells and $n$-cells and since $n-1 \geq 2$ it follows that $X_{1}^{H} \cup W_{1}^{>H}$ is simply connected, and for the same reason $Y_{1}^{H} \cup W_{1}^{>H}$ is simply connected. The pair $\left(W_{1}^{H}, Y_{1}^{H} \cup W_{1}^{>H}\right)$ is $(n-1)$-connected since $W_{1}^{H}$ is obtained from $Y_{1}^{H} \cup W_{1}^{>H}$ by adjoining $n$-cells and similarly $\left(Y_{1}^{H} \cup W_{1}^{>H}\right.$, $\left.X_{1}^{H} \cup W_{1}^{>H}\right)$ is $(n-1)$-connected. Thus by the Hurewicz theorem the homorphisms $\phi$ are isomorphisms and hence by the remarks made at the beginning of the proof each $\phi$ is an isomorphism of $Z\left[G_{\alpha} / H\right]$-modules.

The homology modules are based $Z\left[G_{\alpha} / H\right]$-modules, i.e. free $Z\left[G_{\alpha} / H\right]$ modules together with a family of preferred bases. Thus the homotopy groups are free $Z\left[G_{\alpha} / H\right]$-modules and with corresponding preferred bases given as follows. Let

$$
\begin{gathered}
f_{s}:\left(G / H \times I^{n}, G / H \times \partial I^{n}\right) \rightarrow\left(\bar{b}_{s}^{n}, \dot{b}_{s}^{n}\right) \rightarrow\left(G W_{1}^{H}, G\left(Y_{1}^{H} \cup W_{1}^{>H}\right)\right) \\
h_{s}:\left(G / H \times I^{n-1}, G / H \times \partial I^{n-1}\right) \rightarrow\left(\bar{b}_{s}^{n-1}, \dot{b}_{s}^{n-1}\right) \rightarrow\left(G\left(Y_{1}^{H} \cup W_{1}^{>H}\right), G\left(X_{1}^{H} \cup H_{1}^{>H}\right)\right)
\end{gathered}
$$

be characteristic $G$-maps for $\bar{b}_{s}^{n}$ and $\bar{b}_{s}^{n-1}, s=1, \ldots, m$, as in Corollary 4.4 in Chapter II, and moreover chosen such that

$$
\begin{aligned}
& f_{s}\left(\{e H\} \times I^{n}\right) \subset W_{1}^{H} \\
& h_{s}\left(\{e H\} \times I^{n-1}\right) \subset Y_{1}^{H} \subset Y_{1}^{H} \cup W_{1}^{H} \quad s=1, \ldots, m .
\end{aligned}
$$


Define

$$
\begin{gathered}
\bar{f}_{s}:\left(I^{n}, \partial I^{n}\right) \rightarrow\left(W_{1}^{H}, Y_{1}^{H} \cup W_{1}^{>H}\right) \\
\bar{h}_{s}:\left(I^{n-1}, \partial I^{n-1}\right) \rightarrow\left(Y_{1}^{H} \cup W_{1}^{>H}, X_{1}^{H} \cup W_{1}^{>H}\right)
\end{gathered}
$$

by $\bar{f}_{s}=f_{s} \mid\{e H\} \times I^{n}$ and $\bar{h}_{s}=h_{s} \mid\{e H\} \times I^{n-1}, s=1, \ldots, m$. Since $\tilde{f}_{s}\left(J^{n-1}\right)=\left\{x_{s}\right\}, x_{s} \in X_{1}^{H} \subset X$ and $\bar{h}_{s}\left(\partial I^{n-1}\right)=\left\{\tilde{x}_{s}\right\}, \tilde{x}_{s} \in X_{1}^{H} \subset X$, we have

$$
\begin{gathered}
{\left[\bar{f}_{s}\right] \in \pi_{n}\left(W_{1}^{H}, Y_{1}^{H} \cup W_{1}^{>H}\right),} \\
{\left[\bar{h}_{s}\right] \in \pi_{n-1}\left(Y_{1}^{H} \cup W_{1}^{H}, X_{1}^{H} \cup W_{1}^{>H}\right),}
\end{gathered}
$$

for $s=1, \ldots, m$. The images of the elements $\left[\bar{f}_{s}\right]$ and $\left[\bar{h}_{s}\right], s=1, \ldots$, $m$, under the Hurewicz isomorphisms form preferred bases for the respective homology modules.

Now let

$$
\bar{\partial}\left[\bar{f}_{s}\right]=\sum_{t=1}^{m} a_{s t}\left[\bar{h}_{t}\right], a_{s t} \in Z\left[G_{\alpha} / H\right],
$$

and denote $A=\left(a_{s t}\right)$. Thus we have

$$
\tau(A)=0 \in W h\left(G_{\alpha} / H\right),
$$

and hence the matrix $A$ can be transformed into an identity matrix by a finite sequence of operations of the four types given in $(*)$. By a different choice of characteristic $G$-maps $f_{s}^{\prime}$ for $b_{s}^{n}, s=1, \ldots, m$, (but still satisfying $f_{s}^{\prime}\left(\{e H\} \times I^{n}\right) \subset W_{1}^{H}$ and $\left.\bar{f}_{s}^{\prime}\left(J^{n-1}\right)=\left\{x_{s}^{\prime}\right\}, x_{s}^{\prime} \in X_{1}^{H}\right)$ the matrix of $\bar{\partial}$ can be made into any matrix obtained from $A$ by multiplying rows by \pm elements from $G_{\alpha} / H$.

Now let $1 \leq r \leq m$ and $1 \leq p \leq m$, where $r \neq p$. Let

$$
\bar{v}:\left(I^{n}, \partial I^{n}\right) \rightarrow\left(W_{1}^{-H}, Y_{1}^{H} \cup W_{1}^{>H}\right),
$$

where $\bar{v}\left(J^{n-1}\right)=\{x\}$ for some $x \in X_{1}^{H}$, be such that

$$
[\bar{v}]=\left[\bar{f}_{r}\right]+\left[\bar{f}_{p}\right] \in \tau_{n}\left(W_{1}^{H}, Y_{1}^{H} \cup W_{1}^{>H}\right) \text {. }
$$

Denote $\bar{f}_{p}\left(\stackrel{\circ}{I^{n}}\right)=c_{p}^{n}$. Thus we have $c_{p}^{n} \subset b_{p}^{n} \cap W_{1}^{\mathrm{H}}$ and $\dot{c}_{p}^{n} \subset Y_{1}^{n}$ and moreover $G c_{p}^{n}=b_{p}^{n}$. Since $\bar{f}_{p}: \partial I^{n} \rightarrow Y_{1}^{H} \cup W_{1}^{>H} \cup c_{p}^{n}$ is homotopic to a contant map it follows that the maps

$$
\bar{f}_{r}|, \bar{v}|: \partial I^{n} \rightarrow Y_{1}^{H} \cup W_{1}^{>H} \cup c_{p}^{n}
$$

are homotopic. Define the G-map

$$
v \mid: G / H \times \partial I^{n} \rightarrow G\left(Y_{1}^{H} \cup W_{1}^{>H}\right) \cup b_{p}^{n}
$$

by $(v \mid)(g H, y)=g \bar{v}(y), y \in \partial Y^{n}$. It follows that $v \mid$ is $G$-homotopic to $f_{r} \mid: G / H \times \partial I^{n} \rightarrow G\left(Y_{1}^{H} \cup W_{1}^{>H}\right) \cup b_{p}^{n}$. Now define 


$$
\widetilde{W}=\left(W-b_{r}^{n}\right) \cup_{v \mid}\left(G / H \times I^{n}\right),
$$

(where $v \mid$ is considered as a $G$-map into $W-b_{r}^{n}$ ). By Lemma 4.1 in Chapter II we have $\widetilde{W} \mathrm{~s} W$ rel. $\left(W-b_{r}^{n}\right)$. Moreover the matrix of the boundary homomorphism

$$
\bar{\partial}: \pi_{n}\left(\widetilde{W}_{1}^{H}, Y_{1}^{H} \cup W_{1}^{>H}\right) \rightarrow \pi_{n-1}\left(Y_{1}^{H} \cup W_{1}^{>H}, X_{1}^{H} \cup W_{1}^{>H}\right)
$$

is the one obtained from $A$ by changing the $r$ : th row by adding to it the $p:$ th row.

An expansion of the matrix $A$ to $\left(\begin{array}{cc}A & 0 \\ 0 & 1\end{array}\right)$ is realized geometrically by performing an equivariant elementary expansion of type $H$, that is, adjoin $G / H \times I^{n}$ to $W$ by a $G$-map

$$
\sigma_{+}: G / H \times J^{n-1} \rightarrow G X_{1}^{H} \subset W
$$

defined by $\sigma_{+}(g H, y)=g x$, for some fixed $x \in X_{1}^{H}$.

Thus it now follows that there exists an equivariant $C W$ complex $V$ such that

$$
V \text { s } W \text { rel. } W-\left(\bigcup_{s=1}^{m} b_{s}^{n}\right)
$$

and $(V, X)$ is in simplified form and there are $m+q$, where $q \geq 0$, equivariant $n$-cells $e_{1}^{n}, \ldots, e_{m+q}^{n}$ and $q$ equivariant $(n-1)$-cells $e_{m+1}^{n-1}, \ldots$, $e_{m+q}^{n-1}$ in $V-\left(W-\left(\bigcup_{s=1}^{m} b_{s}^{n}\right)\right)$, and characteristic G-maps $u_{s}: G / H \times I^{n} \rightarrow$ $\bar{e}_{s}^{n}, s=1, \ldots, m+q$, and $h_{s}: G / H \times I^{n-1} \rightarrow \bar{e}_{s}^{n-1}, s=m+1, \ldots$, $m+q$, such that

$$
\bar{\partial}\left[\bar{u}_{s}\right]=\left[\bar{h}_{s}\right], s=1, \ldots, m+q .
$$

Here

$$
\bar{\partial}: \pi_{n}\left(V_{1}^{H}, U_{1}^{H} \cup W_{1}^{>H}\right) \rightarrow \pi_{n-1}\left(U_{1}^{H} \cup W_{1}^{>H}, X_{1}^{H} \cup W_{1}^{>H}\right)
$$

where we have denoted $U=Y \cup e_{m+1}^{n-1} \quad \cup \ldots \cup e_{m+1}^{n-1}$. Observe that $W_{1}^{>H}=V_{1}^{>H}$.

Thus the maps $\bar{u}_{s} \mid, \bar{h}_{s}:\left(I^{n-1}, \partial I^{n-1}\right) \rightarrow\left(U_{1}^{H} \cup W_{1}^{>H}, X_{1}^{H} \cup W_{1}^{>H}\right)$ are homotopic. It follows from this that there exist

$$
\bar{w}_{s}: \partial I^{n} \rightarrow U_{1}^{H} \cup W_{1}^{>H}, s=1, \ldots, m+q,
$$

such that $\bar{w}_{s}$ is homotopic to $\bar{u}_{s} \mid \partial I^{n}$ and $\bar{w}_{s} \mid I^{n-1}=\bar{h}_{s}$, and moreover $\bar{w}_{s}\left(J^{n-1}\right) \subset X_{1}^{H}$. Thus the corresponding $G$-map $w_{s}: G / H \times \partial I^{n} \rightarrow G\left(U_{1}^{H} U\right.$ $W_{1}^{>H}$ is $G$-homotopic to $u_{s} \mid G / H \times \partial I^{n}$. Now form the equivariant $C W$ 
complex $\tilde{V}$ by attaching equivariant $n$-cells $G / H \times I^{n}$ to $V-\left(\bigcup_{s=1}^{m+q} e_{s}^{n}\right)$ by the attaching $G$-maps $w_{s}, s=1, \ldots, m+q$. It follows from Lemma 4.1 in Chapter II that

$$
\tilde{V} \text { s } V \text { rel. } V-\left(\bigcup_{s=1}^{m+q} e_{s}^{n}\right)
$$

Moreover it follows directly from the properties of attaching $G$-maps $w_{s}$ that $\tilde{V}$ collapses equivariantly to $V-\left(\bigcup_{s=1}^{m} b_{s}^{n-1} \cup \bigcup_{s=m}^{m+q} e_{s}^{n-1} \bigcup_{s=1}^{m+q} e_{s}^{n}\right)=$ $W-\left(\bigcup_{s=1}^{m} b^{n-1} \cup \bigcup_{s=1}^{m} b^{n}\right)$

We have shown that

$$
W \mathrm{~s} W-\left(\bigcup_{s=1}^{m} b_{s}^{n-1} \cup \bigcup_{s=1}^{m} b_{s}^{n}\right) \text { rel. } W-\left(\bigcup_{s=1}^{m} b_{s}^{n-1} \cup \bigcup_{s=1}^{m} b_{s}^{n}\right) .
$$

That is, all equivariant cells in $W-X$ which have type $H$ and belong to the $G$-component $G W_{1}^{H}$ have been "removed». Applying this procedure for every subgroup that occurs as the type of some equivariant cell in $W-X$ and to each $G$-component of $W^{K}$ we get

$$
W \text { s } X \text { rel. } X \text {. }
$$

That is $s(W, X)=0 \in W h_{G}(X)$ and we have proved that $\Phi$ is injective.

The surjectivity of $\Phi$ is proved as follows. Let $H$ be a subgroup of $G$ for which $m(H)>0$, and let $G X_{1}^{H}$ be a $G$-component of $X^{H}$. Choose some $x \in\left(X_{1}^{H}\right)^{0}$. Let $G_{\alpha}$ be the group of $X_{1}^{H}$, and let $A=\left(a_{s t}\right)$ be any non-singular $m \times m$ matrix over $Z\left[G_{\alpha} / H\right]$. Now define

$$
h: G / H \times \partial I^{2} \rightarrow G X_{1}^{H} \subset X
$$

to be the $G$-map determined by the condition $(h)\left(\{e H\} \times \partial I^{2}\right)=\{x\}$. Let $G Y_{1}^{H}=G X_{1}^{H} \cup b_{1}^{2} \cup \ldots \cup b_{m}^{2}$ be the equirariant $C W$ complex obtained by adjoining $m$ different equivariant 2-cells $G / H \times I^{2}$ to $G X_{1}^{H}$ by the attaching $G$-map $h$, and let $h_{t}$ denote the corresponding characteristic $G$-map for $b_{t}^{2}$. Then $\left[\bar{h}_{t}\right] \in \tau_{2}\left(Y_{1}^{H}, \mathrm{I}_{1}^{H}\right), t=1, \ldots, m$, form a bases for the free $Z\left[G_{\alpha} / H\right]$-module $\tau_{2}\left(I_{1}^{H}, \mathrm{X}_{1}^{H}\right)$.

Now let $v_{s}:\left(I^{2}, \partial I^{2}\right) \rightarrow\left(Y_{1}^{H}, X_{1}^{H}\right)$ be such that $\left[v_{s}\right]=\sum_{t=1}^{m} a_{s t}\left[\bar{h}_{t}\right]$.

It follows since $\bar{h}_{t}\left(\partial I^{2}\right)=\{x\}$, that we can choose $v_{s}$ such that $v_{s}\left(\partial I^{2}\right)=$ $\{x\}$. Now let $\bar{f}_{s} \mid: \partial I^{3} \rightarrow Y_{1}^{\mathrm{H}}$ be the extension of $v_{s}$ defined by $\left(\bar{f}_{s} \mid\right)\left(J^{n-1}\right)=$ $\{x\}$, and let

$$
f_{s} \mid: G / H \times \partial I^{3} \rightarrow G Y_{1}^{H}
$$


be the corresponding $G$-map. Let $G W_{1}^{H}=G Y_{1}^{H} \cup b_{1}^{3} \cup \ldots \cup b_{m}^{3}$ be obtained by adjoining equivariant 3-cells $G \mid H \times I^{3}$ to $G Y_{1}^{H}$ by $f_{s} \mid, s=$ $1, \ldots, m$, and let $f_{s}$ denote the corresponding characteristic $G$-maps. Now the boundary homomorphism

$$
\bar{\partial}: \pi_{3}\left(W_{1}^{H}, Y_{1}^{H}\right) \rightarrow \pi_{2}\left(Y_{1}^{H}, X_{1}^{H}\right)
$$

is given by $\bar{\partial}\left[\bar{f}_{s}\right]=\sum_{t=1}^{m} a_{s t}\left[\bar{h}_{t}\right]$, i.e. has matrix $A$ in these bases. Thus $\bar{\partial}$ is an isomorphism and it follows easily that $\pi_{n}\left(W_{1}^{H}, X_{1}^{H}\right)=0$ for all $n$. Thus the inclusion $i: X_{1}^{H} \rightarrow W_{1}^{H}$ is a homotopy equivalence and using Corollary 5.5 in Bredon [4] we see that $i: G X_{1}^{H} \rightarrow G W_{1}^{H}$ is a $G$-homotopy equivalence. Thus $X$ is a strong $G$-deformation retract of $G W_{1}^{H} \cup X$ and hence $s\left(G W_{1}^{H} \cup X, X\right) \in W h_{G}(X)$. We now have $\Phi\left(s\left(G W_{1}^{H} \cup X, X\right)\right)=$ $\tau(A) \in W h\left(G_{\alpha} / H\right)$. Since $\Phi$ is a homomorphism this shows that $\Phi$ is surjective.

Known facts about Whitehead groups $W h(\pi)$ together with Theorem 1.4 now gives us the following information about $W h_{G}(X)$.

Theorem 1.5. Let $G$ be a finite abelian group and $X$ an equivariant $C W$ complex such that for any subgroup $H$ of $G$ each component of $X^{H}$ is simply connected. Then $W h_{G}(X)$ is a finitely generated abelian group.

Proof. This follows from Corollary (20.3) in Bass [1] and Theorem 1.4.

Theorem 1.6. Let $G=Z_{m}, m \geq 1$, be a finite cyclic group and let $X$ be as above. Then $W h_{G}(X)$ is a finitely generated free abelian group.

Proof. It is known that if $\pi=Z_{n}, n \geq 1$, then $W h(\pi)$ is a free abelian group on $[n / 2]+1-\delta(n)$ generators, where $[n / 2]$ denotes the integral part of $n / 2$ and $\delta(n)$ is the number of divisors of $n$, see Example 3 on page 54 in Bass [1] and Proposition 4.14 in Bass-Milnor-Serre [3]. Since $\Phi$ is an isomorphism onto a finite direct sum of such Whitehead groups, the theorem follows.

Observe that the result quoted in the above proof implies that $W h\left(Z_{n}\right)=0$ if $n=1,2,3,4$ or 6 , and that for all other finite cyclic groups $\pi$ we have $W h(\pi) \neq 0$. (The fact that $W h\left(Z_{n}\right)=0$ for $n=2,3$ and 4 is due to Higman [8], and the case $n=1$ is elementary.) Since any quotient group of a subgroup of one of the groups $\{e\}, Z_{2}, Z_{3}, Z_{4}$ and $Z_{6}$ is again one of these groups we have.

Theorem 1.8. Let $G=Z_{m}$, where $m=1,2,3,4$ or 6 , and let $X$ be as before. Then $W h_{G}(X)=0$. 
Corollary 1.9. Let $G$ and $X$ be as in Theorem 1.8, and let $Y$ denote an equivariant $C W$ complex. Then any $G$-homotopy equivalence $f: X \rightarrow Y$ is an equivariant simple-homotopy equivalence.

Proof. This follows from Theorem 3.6' in Chapter II and Theorem 1.8.

The case $G=\{e\}$ in Corollary 1.9 is just the standard fact that a homotopy equivalence between simply connected $C W$ complexes is a simple-homotopy equivalence.

Now let $G$ again denote an arbitrary discrete abelian group and let $K$ be a subgroup of $G$. Then the equivariant Whitehead group of the discrete $G$-space $G / K$ is given by

$$
W h_{G}(G / K) \cong \sum_{\text {all }} \oplus W h(K / H) .
$$

Thus in particular

$$
\begin{aligned}
& W h_{G}(G) \cong W h(\{e\})=0, \text { and } \\
& W h_{G}(\{x\}) \cong \sum_{\text {all } H} \oplus W h(G / H) .
\end{aligned}
$$

In many cases it is convenient and natural to restrict the attention to the subgroup of $W h_{G}(X)$ consisting of all elements $s(W, X)$ such that the isotropy groups of points in $W$ belong to some family $F$ of subgroups of $G$. Denote this group by $W h_{G}(X ; F)$. It is clear from the proof of Theorem 1.4 that $\Phi$ gives an isomorphism

$$
W h_{G}(X ; F) \cong \sum_{H \in F} \sum_{i=1}^{m(H)} \oplus W h\left(G_{\alpha(i, H)} / H\right) .
$$

If for example the $G$-action on $X$ is semi-free, that is, the only possible isotropy groups are the trivial group $\{e\}$ and the whole group $G$, then it is in many cases natural to only consider pairs $(W, X)$ where the action on $W$ also is semi-free.

Theorem 1.10. Assume that $X$ is a connected and simply-connected equivariant $\mathrm{CW}$ complex such that the $G$-action is semi-free and each component of the fixed points set is simply connected. Then we have $W h_{G}(X ;\{\{e\}, G\}) \cong W h(G)$.

Corollary 1.11. Let $G=Z \oplus \ldots \oplus Z$ and let $X$ be as in Theorem 1.10. Then $W h_{G}(X ;\{\{e\},\{G\}\})=0$.

Proof. By a Corollary to Theorem 2 in Bass-Heller-Swan [2] we have $W h(Z \oplus \ldots \oplus Z)=0$. The case $W h(Z)=0$ is due to Higman [8].

Corollary 1.12. Let $G=Z \oplus \ldots \oplus Z$ and let $X$ and $Y$ be equivariant $C W$ complexes such that the $G$-action is semi-free and every 
component of the fixed point sets is simply-connected. Then any $G$ homotopy equivalence $f: X \rightarrow Y$ is an equivariant simple-homotopy equivalence.

Proof. By Theorem 3.6. in Chapter II, $f$ is an equivariant simplehomotopy equivalence if and only if $s\left(M_{\tilde{f}}, X\right)=0 \in W h_{G}(X)$, where $M_{\tilde{f}}$ is the mapping cylinder of some equivariant skeletal approximation of $f$. But the $G$-action on $M_{\tilde{f}}$ is semi-free and hence $s\left(M_{\tilde{f}}, X\right)=W h_{G}(X$; $\{\{e\}, G\})=0$.

Both Corollary 1.11 and 1.12 still hold with the family $\{\{e\}, G\}$ replaced by any family $F=\{H\}$ of subgroups of the form $H=$ $H_{1} \oplus \ldots \oplus H_{m}$, where each $H_{i}$ either equals $\{0\}$ or $Z$, here $m$ denotes the number of summands in $G=Z \oplus \ldots \oplus Z$.

Example 1.13. We conclude this section by the following example. Let $G=Z_{5}$ and consider the element $a=\left(-1+t+t^{4}\right) \in Z\left[Z_{5}\right]$. Since $\left(-1+t+t^{4}\right)\left(-1+t^{2}+t^{3}\right)=1$ the element $a$ is a unit and it is known that the $1 \times 1$ matrix [a] represents a generator of $W h\left(Z_{5}\right) \cong$ $Z$. Let $X=\{x\}$ and let $W$ be the equivariant $C W$ complex, constructed as in the proof of the surjectivity of $\Phi$, such that $\Phi(s(W,\{x\}))=\tau[a] \in$ $W h\left(Z_{5}\right)$. Since $s(W,\{x\}) \neq 0 \in W h_{G}(\{x\})$ the inclusion $i:\{x\} \rightarrow W$ is not an equivariant simple-homotopy equivalence. The orbit space $W^{\prime}=$ $Z_{5} \backslash W$ is a $C W$ complex obtained by adjoining a 3 -cell to $S^{2}$ by a degree one map and hence $W^{\prime} \mathrm{s} D^{3}$ rel. $S^{2}$, by Lemma 13 in Whitehead [18]. Thus $W^{\prime} \sim\{x\}$ rel. $\{x\}$ and the induced inclusion on the orbit spaces $i^{\prime}:\{x\} \rightarrow W^{\prime}$ is an ordinary simple-homotopy equivalence. This is the »better example» promised in the discussion preceeding Lemma 1.2 in Chapter II. Observe moreover that $Z_{5}$ acts freely on $W-\{x\}$, and that if we forget the $Z_{5}$-action then the inclusion $i:\{x\} \rightarrow W$ is an ordinary simple-homotopy equivalence.

\section{$\S 2$. Toral actions}

In this section we consider actions by the $n$-dimensional torus $T^{n}, n \geq 1$. (The case $T^{\mathbf{0}}=Z_{2}$ was already treated in Section 1 ).

Theorem 2.1. Let $G=T^{n}, n \geq 1$, and let $X$ be an equivariant $C W^{r}$ complex such that each component of $X^{H}$ is simply connected for every closed subgroup $H$ of $G$. Then we have $W h_{G}(X)=0$.

The proof is similar to the part of the proof of Theorem 1.4 which proves that $\Phi$ is injective. Since $G$ is connected any component of $X^{H}$ is a $G$-component, i.e. the group of every component of $X^{H}$ is $G$. Since 
$G / H$ is connected the group of components of $G / H$ is the trivial group $\{e\}$ and we know that $W h(\{e\})=0$. We omit the details.

Corollary 2.2. Let $G=T^{n}, n \geq 1$, and let $X$ and $Y$ be equivariant $C W$ complexes as in Theorem 2.1. Then any G-homotopy equivalence $f: X \rightarrow Y$ is an equivariant simple-homotopy equivalence.

Proof. Follows from Theorem 3.6' in Chapter II and Theorem 2.1.

By Theorem 2.6 in [9] (or Lemma 4.4 in Matumoto [11]), Corollary 2.2 in particular applies when $X$ and $Y$ are compact differentiable $G$ manifolds. Moreover the assumption that $f: X \rightarrow Y$ is a $G$-homotopy equivalence can by the equivariant Whitehead theorem be expressed in non-equivariant homotopy terms, see Proposition 2.5 in [9].

University of Helsinki,

Department of Mathematics,

SF-00100 Helsinki 10

Finland 


\section{References}

[1] Bass, H.: K-theory and stable algebra. - Inst. Hautes Études Sci. Publ. Math. 22 (1964), 487-544.

[2] -"- Heller, A. and Swan, R. G.: The Whitehead group of a polynomial extension. - Inst. Hautes Études Sci. Publ. Math. 22 (1964), 545-563.

[3] - - Milnor, J. and SerRe, J.-P.: Solution of the congruence subgroup problem for $S L_{n}(n \geqq 3)$ and $S p_{2 n}(n \geqq 2)$. - Inst. Hautes Études Sci. Publ. Math. 33 (1967), $421-499$.

[4] Bredon, G.: Equivariant cohomology theories. - Lecture Notes in Math., Vol. 34, Springer-Verlag, 1967.

[5] Chapman, T.: Hilbert cube manifolds and the invariance of Whitehead torsion. Bull. Amer. Math. Soc. 79 (1973), 52-56.

[6] Cohen, M.: A course in simple-homotopy theory. - Graduate Texts in Math. 10, Springer-Verlag, 1973.

[7] Echmand, B. and Maumary, S.: Le groupe des types simples d'homotopie sur un polyédre, Essays on Topology and Related Topics, Memoires dédiés à Georges de Rham. - Springer-Verlag, 1970.

[8] Higman, G.: The units of group rings. - Proc. London Math. Soc. 46 (1940), $231-248$.

[9] Illman, S.: Equivariant singular homology and cohomology for actions of compact Lie groups. - Proc. Conference on Transformation Groups (University of Massachusetts, Amherst, 1971). Lectures Notes in Math., Vol. 298, Springer-Verlag, 1972, 403-415.

[10] - - Equivariant algebraic topology. - Thesis, Princeton University, Princeton, N. J., 1972.

[11] Matumoto, T.: Equivariant $K$-theory and Fredholm operators. - J. Fac. Sci. Tokyo Sect. I A Math. Vol. 18 (1971), 109-125.

[12] - - On $G-C W$ complexes and a theorem of J.H.C. Whitehead. - J. Fac. Sci. Univ. Tokyo Sect. I A Math. Vol. 18 (1971), 363-374.

[13] Maumary, S.: Type simple d'homotopie (Théorio algébrique), in Torsion et type simple d'homotopie. - Lecture Notes in Math., Vol. 48, SpringerVerlag, 1967.

[14] Mrunor, J.: Whitehead torsion. - Bull. Amer. Math. Soc. 72 (1966), 358-426.

[15] Stöcker, R.: Whiteheadgruppe topologischer Räume. - Invent. Math. 9 (1970), 271-278.

[16] West, J. E.: Mapping cylinders of Hilbert cube factors. - General Topology and Appl. 1 (1971), 111-125.

[17] Whitehead, J. H. C.: Simplicial spaces, nucleii and $m$-groups. - Proc. London Math. Soc. 45 (1939), 243-327.

[18] -»- Simple homotopy types. - Amer. J. Math. 72 (1950), 1-57. 